
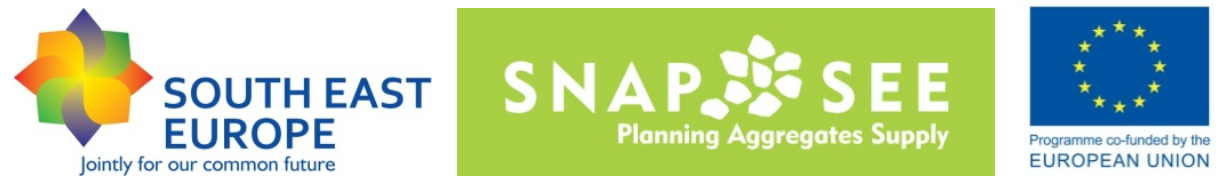

Una Visione delle Migliori Pratiche per la Pianificazione degli Aggregati nel Sud Est Europa

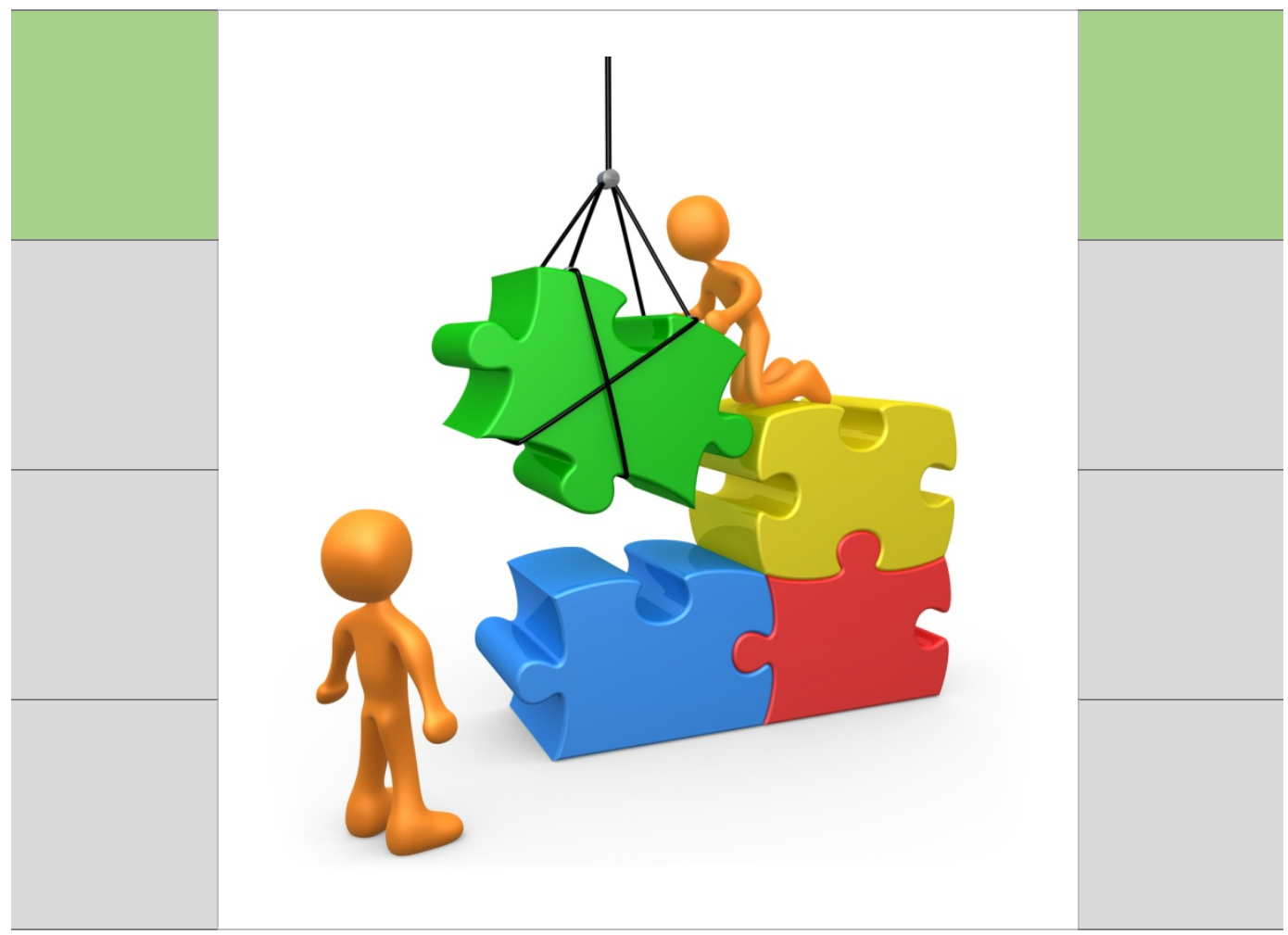

Giugno 2014
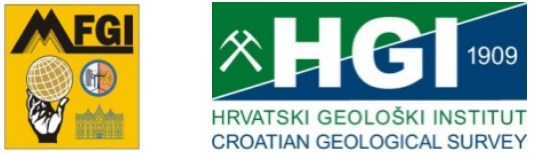



\section{Basata su:}

il lavoro e l'esperienza dei partner di progetto all'interno del Work Package 5 del Progetto SNAP-SEE "Sustainable Aggregates Planning in South East Europe" (SEE/D/0167/2.4/X)

Sito Internet: http://www.snapsee.eu

Risultato Finale dell'Attività 5.3: materiali di supporto per la Pianificazione degli Aggregati compresi l'analisi multisettoriale, la Guida e la Visione Congiunta dei Paesi SEE

\section{Informazioni sulla Pubblicazione}

Revisione \& Layout: $\quad$ Foteini Stathogianni, Urška Dolinar

Design Copertina:

Stelios Mavrigiannakis

Stampa:

Anno di Pubblicazione 2014

\section{Dichiarazione liberatoria:}

Questa pubblicazione SNAP-SEE riflette solamente le opinioni degli autori e l'Autorità Direttiva del Programma di Cooperazione Transnazionale del Sud Est Europa non è responsabile per alcun uso che possa essere fatto delle informazioni contenute all'interno. CSNAP-SEE project 2014 


\begin{tabular}{ll}
\hline Horváth, Zoltán, Dr., MFGI, leader del work package, Ungheria \\
Sári, Katalin, MFGI, Ungheria \\
Müller, Tamás, MFGI, Ungheria \\
Plank, Zsuzsanna, Dr., MFGI, Ungheria \\
Ujházi-Kerék, Barbara, Dr., MFGI, Ungheria
\end{tabular}

\section{Revisori}

\begin{tabular}{ll}
\hline Nome & Affiliazione \\
\hline Shields, Deborah J., Dr. & Università Statale del Colorado, Stati Uniti d'America \\
O'Brien, Jim & Ex Presidente dell'Associazione Europea degli Aggregati, Irlanda \\
Hamor, Tamas Dr. & Ufficio Ungherese per Estrazione Mineraria e Geologia, Ungheria
\end{tabular}

\section{Riconoscimenti}

I partner del progetto SNAP-SEE desiderano ringraziare la Commissione Europea per aver finanziato questo progetto che ha portato ad una cooperazione proficua fra i partner condividendo problematiche similari $e$ una visione comune, e che ha permesso lo sviluppo di questo manuale.

Gli autori desiderano rendere noto il contributo di tutti i partner nella stesura del materiale contenuto in questo manuale.

\section{Digital Object Identifier}

Doi : 10.5474/snapsee-WP5-IT

\section{Citazioni}

Questo manuale dovrebbe venir citato in bibliografia come segue: Horváth, Z., Miko, S., Sári, K. and Dedić, Ž. (2014) A Vision of Best Practices for Aggregates Planning in South East Europe, SNAP-SEE Project, www.snapsee.eu. doi: 10.5474/snapsee-WP5-EN 
$1 \quad$ Informazioni Introduttorie

1.1 L'importanza della Pianificazione degli Aggregati 9

1.2 Le Caratteristiche Speciali della Pianificazione degli Aggregati 10

$1.3 \quad$ L'ambito del Manuale 11

$1.4 \quad$ Struttura del Manuale 11

$2 \quad$ Analisi Multisettoriale sullo Stato della Pianificazione degli Aggregati

2.1 Introduzione 13

$2.2 \quad$ Politiche connesse alla Pianificazione degli Aggregati 14

$2.3 \quad$ Politica di alla Pianificazione degli Aggregati 16

2.3.1 Il quadro normativo e legale in relazione con gli aggregati 17

2.3.2 Vincoli legali contro lo sviluppo della pianificazione degli aggregati 19

2.4 Conclusioni 20

$3 \quad$ Guida sugli Aspetti da considerare nei Piani degli Aggregati 22

3.1 Introduzione 23

3.2 Il contenuto dei documenti di pianificazione e i metodi 24

3.2.1 Perché dovrebbero le autorità fare pianificazione per l'estrazione mi- 25 nerale?

3.2.2 Come dovrebbero le autorità di pianificazione minerale fare pianifica- 26 zione per l'estrazione minerale?

3.2.3 Geologia e potenziale di aggregati 28

3.2.4 Inventario minerali/aggregati 30

3.2.5 Inventario degli aggregati secondari 30

3.2.6 Economia degli aggregati 32

3.2.7 Pianificazione territoriale/urbanistica e impatti ambientali 33

3.2.8 Procedura metodologica - estrazione di materiale grezzo e pianifica- $\quad 35$ zione dell'uso del suolo

3.2.9 Distanze/zone buffer di separazione 37

3.2.10 Analisi di zone di priorità degli aggregati 37

3.2.11 Aspetti Sociali 38

3.2.12 Ripristini, e uso vantaggioso successivo 38 
$4.1 \quad$ Introduzione 41

$4.2 \quad$ Quadro legale normativo $\quad 42$

4.2.1 Livello nazionale 42

4.2.2 Livello regionale e locale 44

4.2.3 Problematiche Europee 44

4.3 Problematiche ambientali 44

$\begin{array}{lll}\text { 4.3.1 Inquinamento dell'aria } & 45\end{array}$

$\begin{array}{lll}4.3 .2 & \text { Rumore } & 45\end{array}$

$\begin{array}{lll}4.3 .3 & \text { Acque } & 45\end{array}$

$\begin{array}{lll}4.3 .4 & \text { Rifiuti minerari } & 46\end{array}$

4.3.5 Biodiversità e compensazione ecologica 46

$\begin{array}{lll}\text { 4.3.6 Ripristini } & 46\end{array}$

$\begin{array}{lll}4.4 & \text { Problematiche sociali } & 47\end{array}$

$\begin{array}{lll}4.5 & \text { Problematiche economiche } & 47\end{array}$

$\begin{array}{lll}\text { 4.5.1 Politiche transnazionali SARM } & 48\end{array}$

$\begin{array}{lll}4.5 .2 & \text { Tassazione } & 48\end{array}$

$\begin{array}{lll}4.6 & \text { Attività professionali } & 49\end{array}$

4.6.1 Inventario 49

4.6.2 Geologia e potenziale degli aggregati 49

$\begin{array}{lll}\text { 4.6.3 Salvaguardia Minerale } & 50\end{array}$

4.7 Tecnologia 50

4.7.1 Recupero/Riciclaggio 50

4.7.2 Esplosivi 51

$\begin{array}{lll}4.7 .3 & \text { Sicurezza } & 51\end{array}$

4.8 Trasporti 51

5 Raccomandazioni sulle Migliori Pratiche nella Pianificazione degli 53 Aggregati

6 Bibliografia 


\section{Premessa}

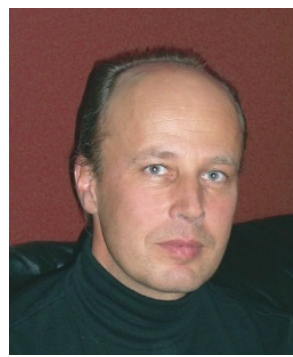

I Paesi del Sud Est Europa (SEE) hanno abbondanti risorse di aggregati per le costruzioni, ovvero ghiaia, sabbia e altri materiali inerti granulari utilizzati nel settore dell'edilizia. Tuttavia, vi è la necessità di guardare nella direzione di una gestione sostenibile della risorsa degli aggregati (SARM sustainable aggregate resource management) e considerare un mix di fornitura sostenibile (SSM - sustainable supply mix) di aggregati a livello regionale per migliorare l'efficienza dell'uso della risorsa e supportare lo sviluppo sostenibile. SARM rappresenta l'estrazione mineraria e la gestione dei rifiuti efficiente con basso impatto socio-ambientale, accompagnata da un ampio coinvolgimento dei soggetti interessati. II mix SSM è costituito da aggregati da fonti multiple, comprendendo anche i rifiuti riciclati e i sottoprodotti industriali (scorie), così come i materiali primari prodotti sia a livello locale sia quelli importati, che tutti insieme massimizzano il beneficio netto della fornitura degli aggregati nel tempo.

L'implementazione di SARM e SSM richiede una politica di supporto e una struttura di pianificazione territoriale. Purtroppo, a causa delle differenze regionali e del (diverso) sviluppo storico, gli approcci a politiche, a pianificazioni e a gestioni sugli aggregati differiscono tra i Paesi SEE e in alcuni casi sono completamente diverse. I temi trattati sono distribuiti all'interno di differenti documenti delle amministrazioni rendendo difficile il coordinamento ed una comprensione globale, ed includono politiche e piani che si occupano di gestione e fornitura degli aggregati. Vi è quindi una quasi totale mancanza di coordinamento sulla pianificazione per la fornitura da aggregati primari e secondari. I Paesi SEE hanno dati insufficienti per sostenere la pianificazione degli aggregati, inadeguata capacità e competenza per affrontare una pianificazione sia degli aggregati primari che secondari, e un'insufficiente partecipazione dei portatori di interesse nello sviluppo dei piani di gestione degli aggregati.

Lo scopo del progetto "Pianificazione Sostenibile degli Aggregati nel Sud Est Europa" (SNAP-SEE) era di creare e promulgare uno strumento per la pianificazione degli aggregati per aiutare i governi SEE a migliorare i loro processi di pianificazione e gestione degli aggregati. E' basato sui risultati del progetto SARMa - Gestione Sostenibile delle Risorse di Aggregati - un precedente progetto finanziato dal fondo di Cooperazione Transnazionale SEE (http:// www.sarma.eu). SNAP-SEE è stato finanziato dal Programma di Cooperazione Transnazionale della UE Sud Est Europa (SEE) e aveva 27 partner da 12 Paesi SEE e (oltre) la Turchia. L'università di Leoben, in 
Austria, era il partner leader. II progetto SNAP-SEE aveva durata 2 anni che sono terminati a settembre 2014.

Lo strumento SNAP-SEE per la Pianificazione degli Aggregati comprende 4 prodotti che sono correlati fra loro.

\section{Buone norme nella pianificazione sostenibile degli aggregati}

Questo documento presenta una Visione per una transizione alla pianificazione integrata, esauriente e sostenibile nei Paesi SEE. Contiene le spiegazioni delle problematiche che hanno bisogno di essere affrontate, fasi provvisorie che possono essere compiute verso una pianificazione più sostenibile, ed un esame delle componenti che un piano sostenibile dovrebbe contenere.

2. Come costruire un piano sostenibile degli aggregati

Questo documento rappresenta una linea guida per la pianificazione, contenente le spiegazioni del processo pianificatorio stesso e le sue varie fasi. Sono forniti esempi di pianificazione ben redatta che incarnano i principi, gli approcci e le azioni necessarie per raggiungere gli obiettivi della Visione delineati nel report delle buone norme.

3. Consultazione in supporto alle buone norme nella pianificazione sostenibile degli aggregati

Questo documento fornisce una guida passo-passo su come programmare e condurre una consultazione delle parti interessate così come assicurare che le associazioni industriali, governative e non e anche la società civile possano partecipare e dare il loro apporto nel processo pianificatorio. Vengono forniti gli strumenti per la conduzione delle consultazioni.

4. Dati ed analisi dei dati in supporto alle buone norme nella pianificazione sostenibile degli aggregati

Questo documento tratta di vari tipi di dati che forniscono il background informativo essenziale per il processo di pianificazione. I temi affrontati sono la definizione, la significatività, la disponibilità, l'organizzazione dei dati. Si presentano quindi i metodi per la validazione e l'analisi dei dati, considerando anche gli approcci alla previsione di domanda.

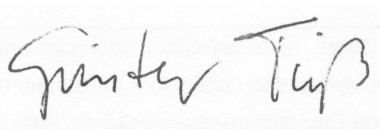

Coordinatore del Progetto Guenter Tiess

Montanuniversität Leoben (Università di Leoben) 
Un processo di pianificazione che con chiarezza tratta $i$ bisogni sociali all'interno di uno Stato o regione, è sia un qualcosa di complesso che di essenziale. Pertanto, l'Autorità Competente ha bisogno di sufficienti informazioni relative alla situazione del settore di pianificazione sia per gli aggregati primari che secondari, e relative alle migliori metodologie di pianificazione e ad una guida che conduca alla gestione sostenibile delle risorse minerali/di aggregati. Perché si compia una pianificazione esauriente ed integrata, si deve determinare il livello e gli obiettivi di tutti $i$ testi relativi alla pianificazione degli aggregati, così come la relazione di ogni modulo della pianificazione ed a quale grado tutto ciò contribuisca all'efficienza della risorsa e alla sostenibilità.

Le lacune identificate nella pianificazione, l'eccellenze, le sovrapposizione o contrasti fra $i$ settori, $i$ fabbisogni e le preferenze sia per gli aggregati primari che secondari sono problematiche importanti durante l'analisi dei cicli di pianificazione in ogni Paese.

Basandosi sulla presentazione dello stato dell'arte della pianificazione in ogni Paese partner in SEE, questo Manuale contribuisce ad una pianificazione più consistente, integrata ed efficiente nell'uso delle risorse a livello nazionale/regionale, la guida migliore per una pianificazione sostenibile degli aggregati e la visione congiunta comprendente gli argomenti principali che dovrebbero essere sviluppate nel prossimo futuro. 
1. Informazioni introduttorie

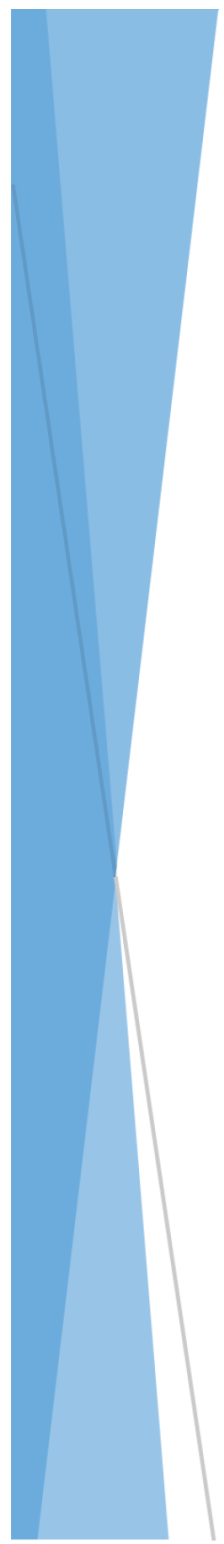




\subsection{L'importanza della Pianificazione degli Aggregati}

Gli aggregate primari sono principalmente sabbia, ghiaia, massi frantumati; gli aggregati secondari si possono recuperare da rifiuti da costruzione e demolizione, aggregati industriali, materiali scavati in opere civili, ecc. (Glossario SARMa). Basandosi sui dati UEPG (Tiess 2010) sulla crescita economica, in futuro sarà richiesto nel Sud Est Europa un maggior quantitativo di aggregati. Gli aggregati hanno un'importanza essenziale per la crescita economica. Sebbene le risorse di aggregati sembrino essere illimitate (la maggioranza dei depositi sono noti), il loro accesso sta divenendo una sempre più crescente difficoltà. Non solo aree protette (es. siti di conservazione della biodiversità e patrimoni culturali) e attitudini sociali, ma anche lo sviluppo di edifici e strade ostacolano l'accesso alle risorse di aggregati occupando e inibendo le aree con potenziale estrattivo di risorse. Pertanto, la pianificazione della fornitura di aggregati è essenziale.

Anche se i fabbisogni sociali di aggregati sono soddisfatti dal settore privato, l'Autorità Competente è responsabile di controllare le proprie fonti attraverso la pianificazione degli aggregati, che fa riferimento ad una varietà di politiche intesa ad assicurare una fornitura sicura di aggregati necessaria per tutto ciò che venga costruito in un Paese o regione, dagli edifici alle infrastrutture. L'Autorità Competente ha, o dovrebbe acquisire, tutte le informazioni necessarie sulle risorse nazionali di aggregati primari e secondari, e gestire questi inventari. La legislazione e la determinazione delle future strategie per lo sviluppo cadono quindi anche fra le competenza dell'Autorità Competente. Pertanto, usando tutte queste informazioni, un'esauriente pianificazione degli aggregati può essere solo sviluppata dall'Autorità Competente, e dovrebbe essere armonizzata a livello statale, regionale e locale.

Il sistema di pianificazione ha un ruolo fondamentale nel fornire una quadro all'interno del quale decisioni solide e consistenti possono venire prese sulle proposte di sviluppo minerario. I piani minerari, specialmente quando sono integrati nei piani territoriali di uso del suolo, aiutano l'industria e le autorità pertinenti a stabilire una quadro di pianificazione stabile per l'estrazione dei minerali per un lungo periodo. Sono quindi anche molto efficaci a creare una più integrata strategia di sviluppo sostenibile che tenga in considerazione un più ampio spettro di interessi sociali, come anche la conservazione naturale, in una fase molto precoce del processo decisionale. Quindi, le Autorità dovrebbero cercare attraverso le loro politiche di piani di sviluppo e le loro decisioni di tenere conto di tutti i costi e benefici associati con l'estrazione minerale secondo i principi dello sviluppo sostenibile. Gli scopi principali che si riferiscono alla pianificazione minerale sono i seguenti:

$\checkmark$ Progresso sociale che riconosce i bisogni di ciascuno: per provvedere ai benefici sociali di una accresciuta prosperità attraverso una fornitura adeguata 
di minerali di cui la società ha bisogno ora e in futuro, insieme con servizi di protezione e miglioramento;

$\checkmark \quad$ Una protezione efficace dell'ambiente: per proteggere cose che sono altamente preziose per le loro qualità intrinseche, come la fauna selvatica, i paesaggi e il patrimonio storico; e per proteggere la salute e la sicurezza umana controllando che gli impatti ambientali causati dall'estrazione e trasporto minerari siano entro limiti accettabili; e per assicurare, senza compromessi, il ripristino e la cura-dopo-ripristino per provvedere ad un uso successivo appropriato e vantaggioso;

$\checkmark \quad$ Uso prudente delle risorse naturali: per aiutar a conservare le risorse non rinnovabili per le generazioni future attraverso l'uso efficiente, il recupero e la minimizzazione dei rifiuti; per proteggere le risorse rinnovabili da danni seri o inquinamento; e per promuovere l'uso di materiali alternativi appropriati;

$\checkmark \quad$ Il mantenimento di livelli elevati e stabili di crescita economica: per controllare una fornitura adeguata di minerali che sono necessari a prezzi ragionevoli; salvaguardare le risorse per le future generazioni.

\subsection{Le Caratteristiche Speciali della Pianificazione degli Aggregati}

La pianificazione per la fornitura di minerali ha un numero di caratteristiche speciali che non sono presenti in altri piani (http://planningguidance.planningportal.gov.uk):

$\checkmark \quad$ I minerali si possono lavorare (cioè estrarre) solamente dove sono presenti, così le alternative di localizzazione per l'estrazione dei minerali economicamente fattibile e accettabile dal punto di vista ambientale possono essere limitate. Ciò significa che è necessario considerare la protezione dei minerali da piani non minerali e che comporta implicazioni per la preparazione di piani minerali e l'approvazione di piani non minerali in aree definite di salvaguardia minerale;

$\checkmark \quad$ L'estrazione è un uso temporaneo del territorio, sebbene spesso abbia luogo per un lungo periodo di tempo;

$\checkmark \quad$ L'estrazione può avere effetti ambientali sfavorevoli e positivi, ma alcuni effetti negativi possono venire efficacemente mitigati;

$\checkmark \quad$ Finché l'estrazione è un continuo processo di sviluppo, vi è l'esigenza ad un costante monitoraggio, e se necessario, ad un'esecuzione di assicurare la conformità con condizioni che sono necessarie a mitigare gli impatti delle operazioni di estrazione dei minerali;

$\checkmark$ Successivamente all'estrazione, il terreno dovrebbe venire ripristinato per renderlo adatto a usi futuri vantaggiosi. 


\subsection{L'ambito del Manuale}

Le politiche di pianificazione degli aggregati sono obiettivi governativi dichiarati nel campo degli aggregati; la pianificazione è la creazione di procedure formali da seguire per raggiungere gli obiettivi; e la gestione è l'amministrazione dei piani. Il progetto SNAP-SEE - Sustainable Aggregates Planning in South East Europe (SEE) - Pianificazione Sostenibile degli Aggregati nel Sud Est Europa (SEE) affronta una questione chiave: come possono i Paesi e le regioni SEE migliorare i processi di pianificazione degli aggregati, integrare la pianificazione per gli aggregati primari e secondari per aumentare l'efficienza delle risorse, e alzare i livelli di competenza fra le Autorità, l'industria e la società civile per quel che riguarda la gestione, la pianificazione e la fornitura degli aggregati.

Pertanto, lo stato della pianificazione, le migliori pratiche nella pianificazione (guida) e gli argomenti principali che dovrebbero venire inclusi, ovvero, la visione congiunta, sono fondamentali per migliorare le politiche e il quadro normativo e, di conseguenza, la pianificazione sostenibile degli aggregati.

\subsection{Struttura del Manuale}

II manuale è organizzato come segue. II capitolo 2 introduce i risultati dell'analisi multisettoriale dello stato della pianificazione, prima discutendo tutte le relative politiche, di seguito la pianificazione degli aggregati, il loro quadro normativo e i vincoli legali.

Il capitolo 3 è una guida che mostra come rendere migliore la pianificazione. Questo capitolo fornisce informazioni generali sulla pianificazione minerale e i documenti (base di conoscenza, domanda di aggregati, fornitura di aggregati di potenziale, impatti, analisi del ciclo di vita) e presenta il contenuto di documenti di pianificazione e metodi (autorità di pianificazione, potenziale di aggregati, inventario, economia di aggregati, pianificazione territoriale, aspetti sociali, ripristini ecc..).

Il capitolo 4 contiene una visione congiunta per il Sud Est Europa riguardante la Pianificazione degli aggregati. Un quadro normativo ideale, futuro è introdotto a scala nazionale ed europea. In seguito le relative problematiche ambientali (inquinamento dell'aria, del rumore, delle acque, rifiuti minerari, biodiversità e ripristini), sociali ed economici (politica transnazionale SARM, tassazione), compiti professionali (inventario, potenziale aggregato, salvaguardia minerale) sono discusse. Infine la tecnologia (recupero/riciclo, l'uso di esplosivi, sicurezza) e il trasporto è presentato.

Il capitolo 5 riassume le raccomandazioni più importanti riguardanti la pianificazione degli aggregati. 


\section{Analisi Multisettoriale sullo Stato della Pianificazione degli Aggregati}

L'obiettivo dell'Analisi Multisettoriale è di determinare come la pianificazione viene condotta nei Paesi partner, comprendendo a quale punto ogni Paese partner si trovi nel ciclo di pianificazione. La pianificazione della fornitura degli aggregati è un'attività governativa, il cui scopo è di determinare le politiche, il quadro legale, le azioni, e le informazioni che saranno necessarie per assicurare la disponibilità di quantitativi adeguati di aggregati primari e secondari per le economie nazionali/regionali nel breve, medio e lungo termine. La pianificazione richiede considerazioni sulla domanda attesa, sulle fonti di approvvigionamento e sui vincoli a ciò, sulla politica attuale, sul quadro normativo 


\subsection{Introduzione}

L'analisi multisettoriale riguardante gli aggregati è necessaria a causa della complessità delle pratiche di pianificazione delle differenti autorità. $E^{\prime}$ un metodo per rivelare dove e come gli aggregati sono menzionati nei documenti ufficiali che rappresentano differenti settori, che potrebbero avere un impatto sull'approvvigionamento degli aggregati.

I settori in relazione con gli aggregati sono:

$\checkmark$ Gestione mineraria/minerale

$\checkmark$ Ambiente, comprese la gestione delle acque e la conservazione della natura

$\checkmark$ Acquisti Verdi (settore pubblico)

$\checkmark$ Gestione dei rifiuti

$\checkmark$ Recupero/riciclaggio

$\checkmark \quad$ La pianificazione dell'uso del suolo

La Figura 2.1 illustra i settori in relazione con le politiche di pianificazione degli aggregati

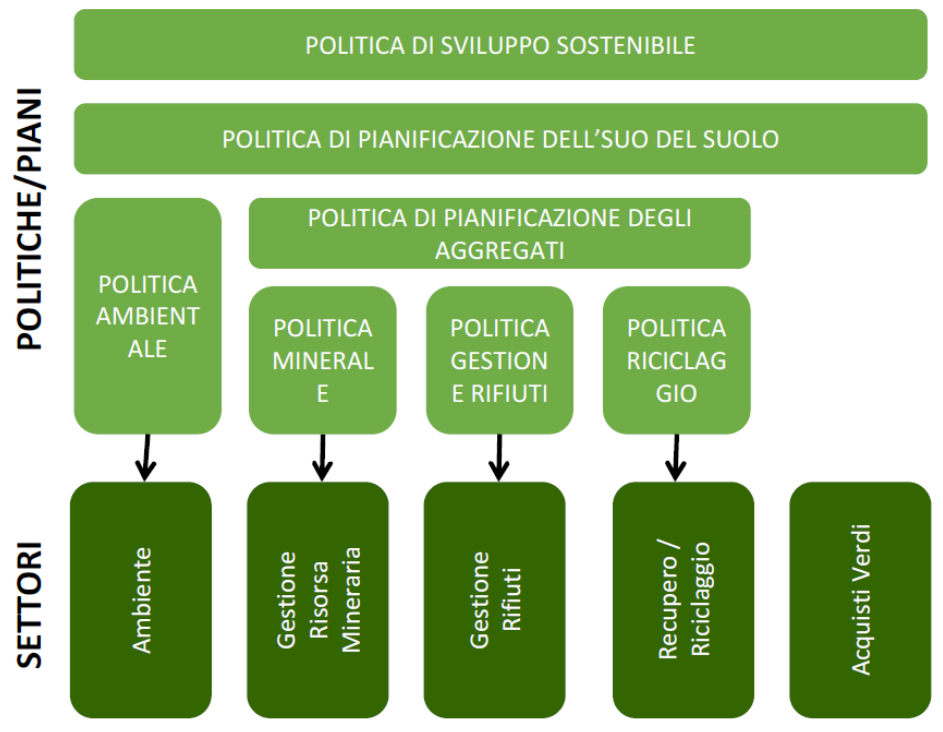

Figura 2.1. Settori in relazione con le politiche di pianificazione degli aggregati 
Questi settori sono inclusi in norme e politiche, come la politica di sviluppo sostenibile, quella minerale, di uso del suolo, di gestione dei rifiuti, di riciclaggio/recupero e la politica ambientale. La pianificazione dell'uso del suolo e la politica di sviluppo sostenibile coprono svariati settori. Una pianificazione sostenibile degli aggregati gestisce complessivamente sia quelli primari (da risorsa minerale) che secondari (da rifiuti e sottoprodotti).

\subsection{Politiche connesse alla Pianificazione degli Aggregati}

Questa analisi multisettoriale in Error! Reference source not found. presenta lo stato dell'arte della pianificazione degli aggregati in Albania (AL), Austria (AT), Cantone Bosnia-Erzegovina (Bosnia-Herzegovina; BA), Croazia (HR), Grecia (GR), Ungheria (HU), Regione Emilia-Romagna (IT-ER) e Provincia Autonoma di Trento (IT-T) (Italia), Montenegro (ME), Romania (RO), Serbia (RS), Slovacchia (SK), Slovenia (SI) e Turchia (TR). Molti Paesi e regioni SEE si basano sullo sviluppo sostenibile, includendo politiche minerali, quadri di pianificazione dell'uso del suolo, politiche di gestione dei rifiuti e ambientali. Tuttavia, solo alcuni tengono conto di politiche di recupero/riciclaggio e di pianificazione degli aggregati.

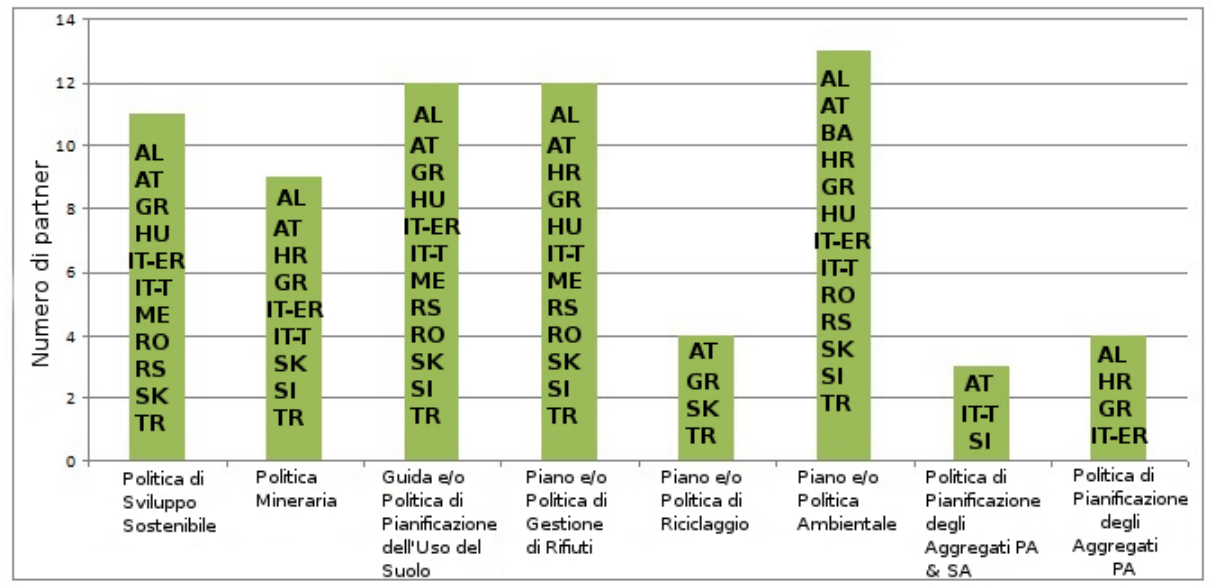

Figura 2.2 Illustrazione della Politica di Sviluppo Sostenibile, Politica Minerale, Politica di Pianificazione degli Usi del Suolo, Politica di Gestione dei Rifiuti, Politica di Recupero/Riciclaggio, Politica Ambientale e Politica di Pianificazione degli Aggregati nei Paesi SEE. PA: Primari Aggregati; SA: Secondari Aggregati

Undici Paesi o regioni hanno Politiche di Sviluppo Sostenibile. Sette si riferiscono ai minerali tuttavia dichiarano soprattutto obiettivi generali come la gestione razionale minerale e l'efficienza della risorsa. Cinque partner si riferiscono agli aggregati e otto Paesi o regioni al recupero/riciclaggio nella loro Politica di Sviluppo Sostenibile e solo quattro partner considerano gli acquisti verdi. Molte Politiche di Sviluppo Sostenibile 
dei partner nominano una gestione razionale e sostenibile degli aggregati relativamente a tutti i tipi di minerali ma solo in termini generali.

Nove Paesi o regioni hanno Politiche Minerali; tutti gli altri partner si riferiscono agli aggregati nelle loro Politiche Minerali (Figura 2.3). Otto Paesi/regioni partner si occupano di pianificazione dell'uso del suolo. La Grecia, la Slovacchia e la Slovenia considerano il riciclaggio/ recupero; Albania, Grecia e Slovacchia considerano la gestione dei rifiuti.

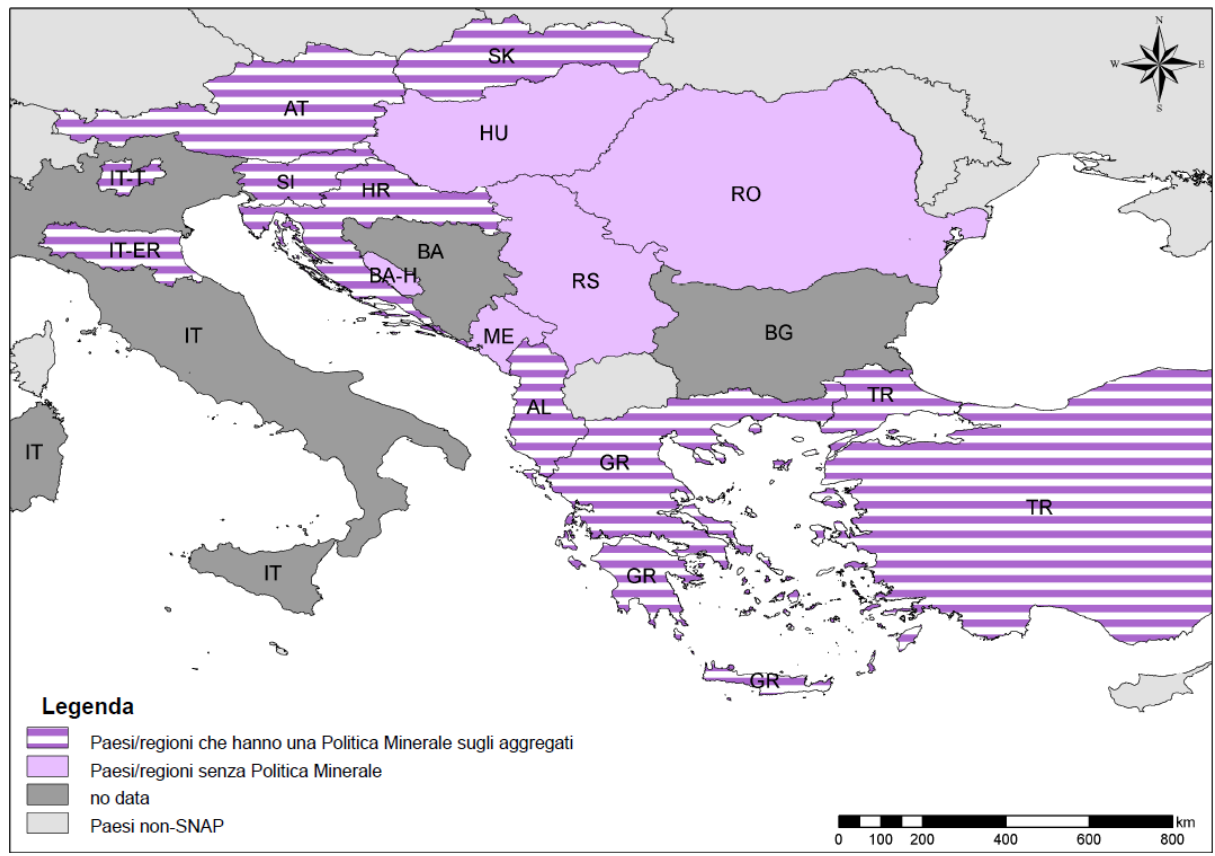

Figura 2.3. Politica Minerale in Sud Est Europa

Dodici Paesi o regioni hanno Politiche di pianificazione di Uso del suolo. Dieci si assicurano l'accessibilità dei minerali, la protezione e l'uso sostenibile. Otto si riferiscono soprattutto agli aggregati. Quattro Paesi menzionano il recupero/riciclaggio nelle loro Politiche di Pianificazione di Uso del Suolo. Solo l'Austria considera gli Acquisti Verdi.

Tutti i partner, eccetto il Cantone Bosnia-Erzegovina e la Regione Emilia-Romagna, hanno Politiche di Gestione dei Rifiuti, sebbene la Politica di Gestione dei Rifiuti nella Regione Emilia-Romagna sia in fase di completamento. Dieci Paesi fanno riferimento al recupero. Sette considerano i minerali e cinque partner si occupano di aggregati esplicitamente. Comunque, le Gestioni dei Rifiuti ungheresi e montenegrine non fanno riferimento a nessun tipo di aggregato, solo ai rifiuti da C\&D. Quattro partner no- 
minano gli acquisti verdi nelle loro Politiche di Gestione dei Rifiuti. Solo la Grecia si riferisce al Rifiuto Zero. Dieci Paesi o regioni hanno adottato la legislazione della Commissione Europea, cosicché la loro Gestione dei Rifiuti comprende un'azione incentivante per il recupero. Solo quattro Paesi hanno Politiche di Recupero separate dalle Politiche di Gestione dei Rifiuti.

Tutti i partner, eccetto il Montenegro, hanno Politiche Ambientali. Sette Paesi considerano i minerali e il recupero nelle loro Politiche Ambientali, nonostante nelle Politiche Ambientali ungheresi e serbe il recupero sia considerato indipendentemente dagli aggregati. Sei partner si occupano di aggregati nelle loro Politiche Ambientali. Austria, Ungheria e Regione Emilia-Romagna nominano gli Acquisti Verdi.

Trattare di potenziali materie prime secondarie (es. rifiuti minerari, rifiuti da C\&D, impianti di produzione d'energia elettrica, impianti d'incenerimento, ceneri da fonderia, scorie) non è omogeneo nella macroregione SEE. In Slovenia il recupero è vincolante dal punto di vista legale. In Ungheria è promosso ma non sempre obbligatorio; raccolta separata e smaltimento sono anche regolamentate. In Montenegro il recupero non è incluso nella legge, ma la gestione dei rifiuti estrattivi appartiene all'attività mineraria. In Serbia quasi tutti i tipi di materiali primi secondi sono smaltiti in discarica. Nella pratica i materiali grezzi secondari sono smaltiti (Serbia), raccolti separatamente e riusati (Provincia Autonoma di Trento, Slovenia, Austria) o trattati combinati (Ungheria, Croazia, Montenegro, Albania).

\subsection{Politica di Pianificazione degli Aggregati}

Solo tre partner (Austria, Slovenia e Provincia Autonoma di Trento) hanno Politiche di Pianificazione degli Aggregati che considerano sia gli aggregati primari e secondari. In altri quattro Paesi o regioni (Albania, Croazia, Grecia e Regione Emilia-Romagna) vi sono Politiche di Aggregati che considerano solo quelli primari (Figura 2.4).

Le autorità responsabili per la pianificazione degli aggregati sono minerarie, pianificazione dell'uso del suolo e altre autorità come quelle economiche, responsabili della sostenibilità, della gestione delle acque e governi locali e regionali. In tutti i Paesi dove la pianificazione degli aggregati esiste, è stata creata attraverso il coinvolgimento dei soggetti interessati (governi locali, enti pubblici e privati). Queste politiche sono riviste regolarmente (in Provincia Autonoma di Trento ogni 10 anni, in Croazia 1012 anni); dipende dalle necessità e dal tipo di documento.

Alcune Paesi (Austria, Croazia, Slovenia e la Provincia Autonoma di Trento) si occupano del bilancio fornitura-domanda; la domanda futura è principalmente stimata sulla base della produzione recente e sui piani di sviluppo dei futuri usi del suolo. 
In termini di valutazione dell'estrazione degli aggregati e altri (potenzialmente influenzati) diritti di utilizzo sono usati principalmente i dati GIS. Per esempio, i dati sulla morfologia, infrastrutture, posizione delle cave, aree protette (naturali o patrimoni culturali) e sottoposte a rischio idrogeologico e ambientale sono disponibili in formato GIS. I dati statistici sono quindi utilizzati nella Provincia Autonoma di Trento, in Croazia ed Austria (es. produzione, consumo, tipo, recupero).

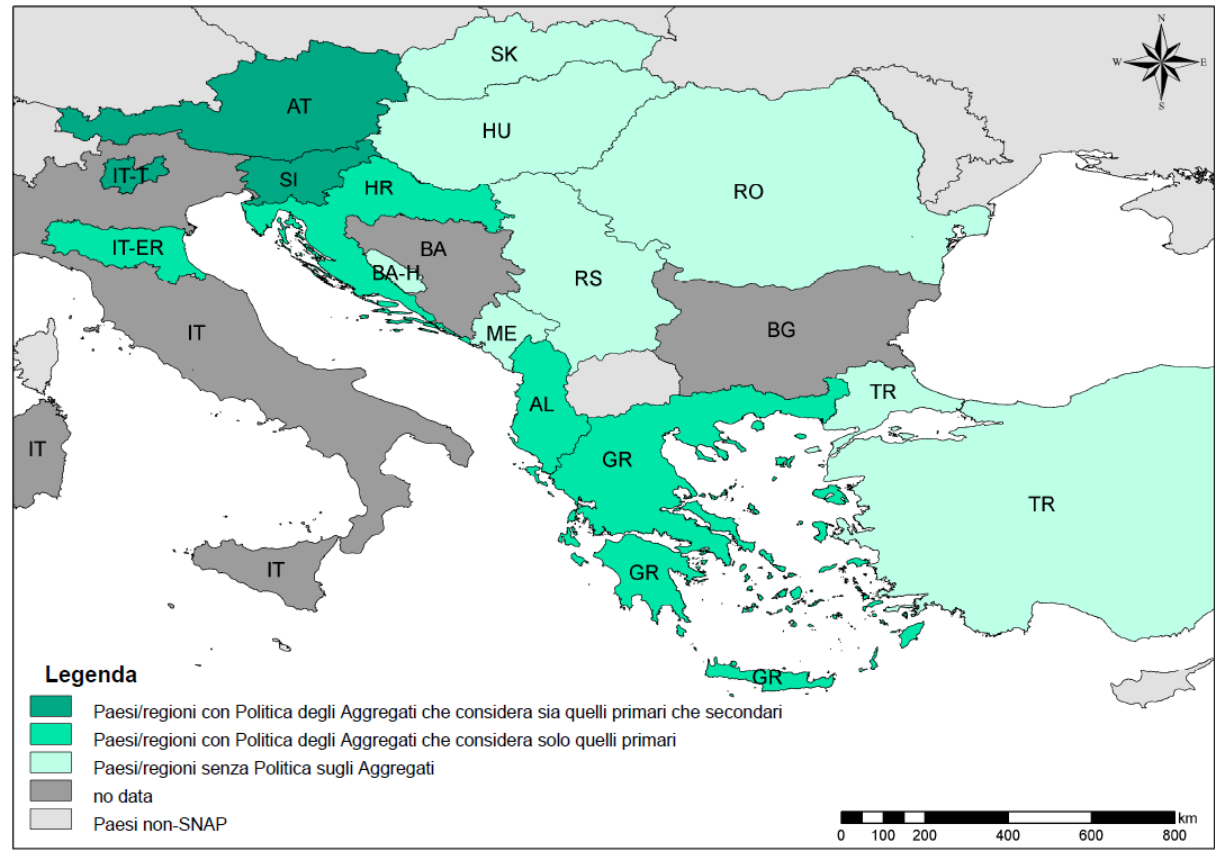

Figura 2.4. Politica di Pianificazione degli Aggregati nel Sud Est Europa

I piani degli aggregati, integrati nel quadro di pianificazione dell'uso del suolo, affrontano molti diversi argomenti, es. la conservazione della natura, gli impatti sociali, il contributo economico, il trasporto, il post-trattamento ecc. Nella Provincia Autonoma di Trento e nella Regione Emilia-Romagna la protezione ambientale ha grande importanza. In Slovenia diversi argomenti come la caccia, l'accudire le arnie e l'educazione sono pure compresi. Tutte le politiche di pianificazione degli aggregati si riferiscono ad altre leggi, es. la pianificazione dell'uso del suolo, il piano rifiuti, la politica ambientale e le normative sul trasporto.

\subsubsection{II quadro normativo e legale in relazione con gli aggregati}

Secondo i risultati del report del progetto SARMA (Hámor 2011) il proprietario dell'estrazione degli aggregati (cioè l'attività mineraria) è principalmente lo Stato; in 
Austria, Grecia, Slovacchia, Regione Emilia-Romagna e in Turchia il proprietario del terreno; e nella Provincia Autonoma di Trento entrambi (Figura 2.5).

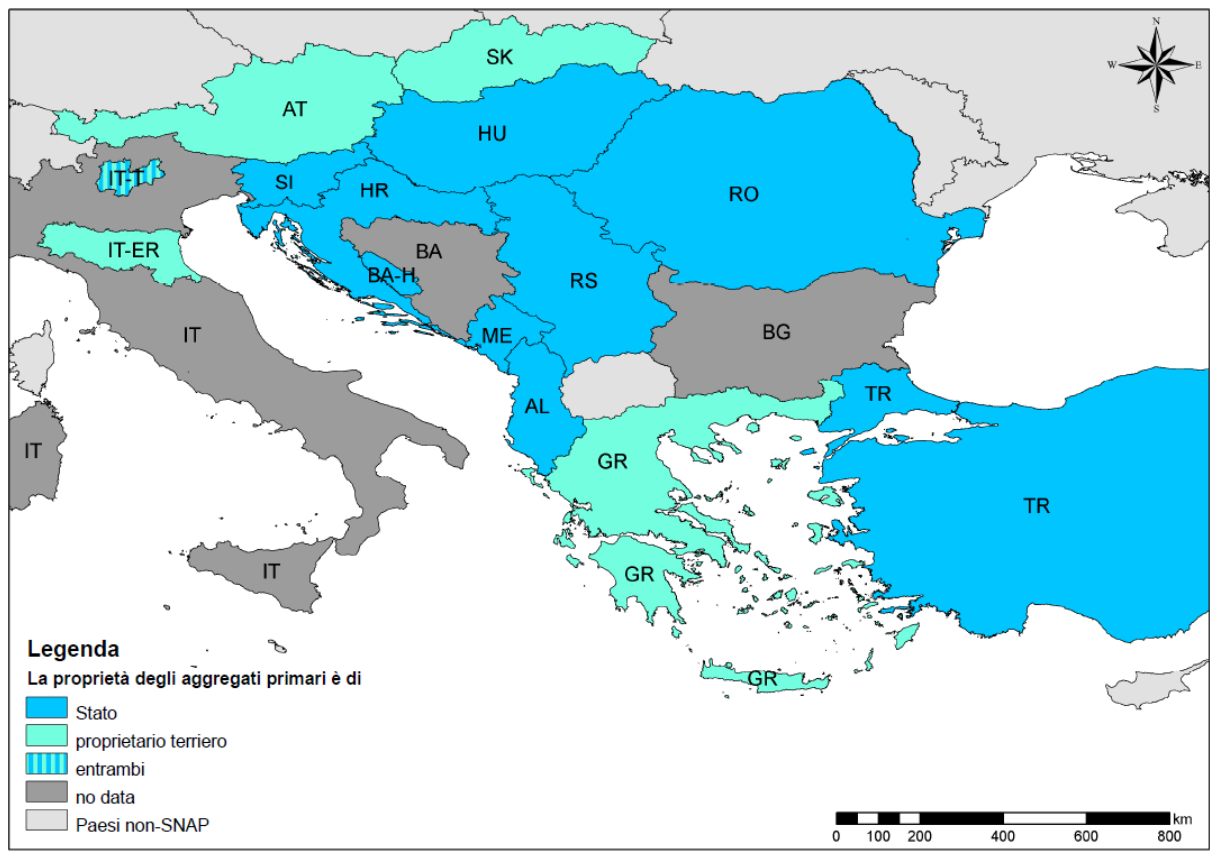

Figura 2.5. La proprietà degli aggregati primari nel Sud Est Europa

L'autorità mineraria (e geologica) gioca un ruolo importante nelle fasi di autorizzazione relative alla pianificazione degli aggregati. In molti Paesi l'autorità ambientale, il governo locale, la pianificazione dell'uso del suolo e altre autorità sono quindi responsabili per l'autorizzazione.

In tutti i Paesi partner le problematiche di protezione della natura sono affrontate ed incorporate nelle autorizzazioni ambientali considerando le problematiche degli aggregati. Eccetto la Romania e la Turchia, in tutti i Paesi la trasposizione di questa legislazione conduce alla designazione di aree di assoluto divieto per l'estrazione degli aggregati.

La partecipazione pubblica è assicurata durante la fase autorizzativa in tutti i Paesi; in Albania attraverso audizioni pubbliche, in Italia e Turchia in forma scritta, mentre negli altri Paesi SEE si usano entrambi i metodi. II modello autorizzativo non è omogeneo nei Paesi SEE, è principalmente uno sportello unico o misto, ma esiste anche il modello di valutazione parallela (Figura 2.6). 


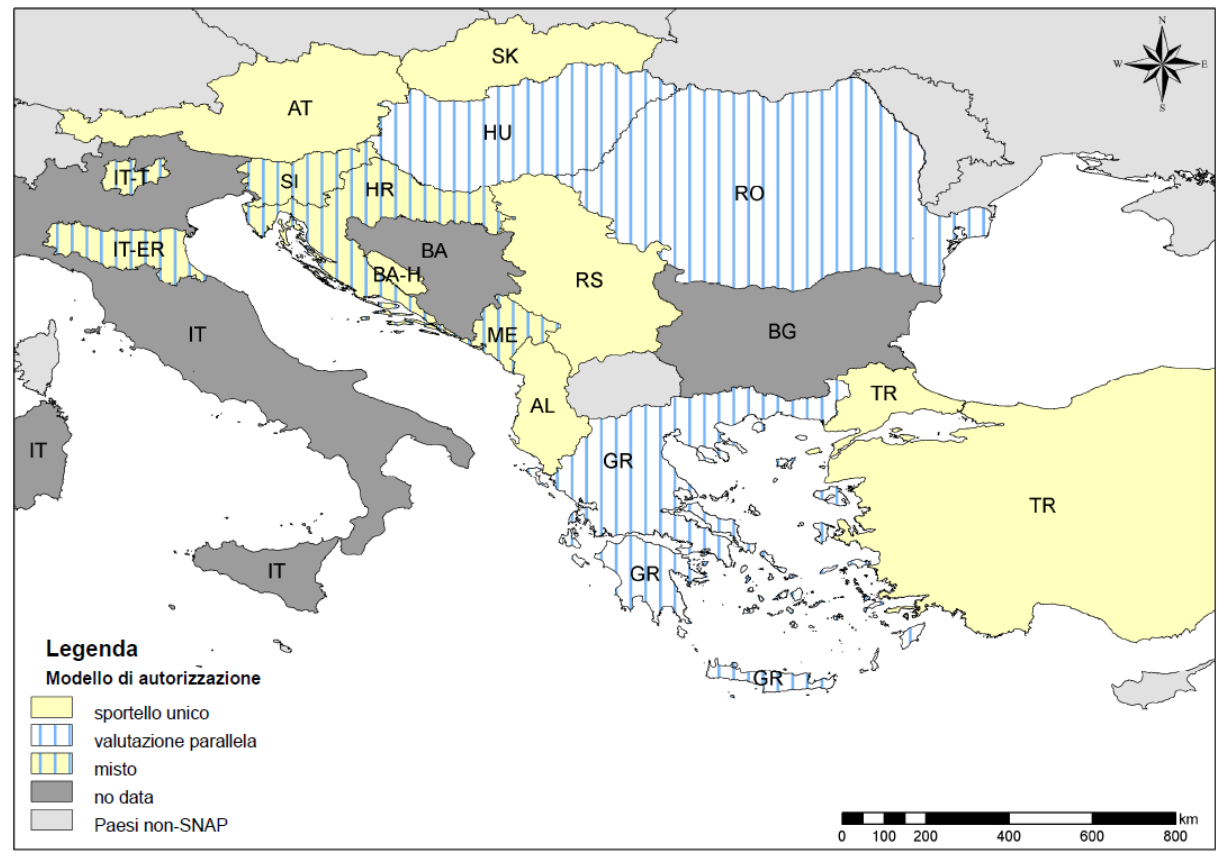

Figura 2.6. Modello di autorizzazione nel Sud Est Europa

\subsubsection{Vincoli legali contro lo sviluppo della pianificazione degli aggregati}

In differenti Paesi SEE vi sono differenti vincoli legali e normativi relativi allo sviluppo della pianificazione degli aggregati. La mancanza di termini omogenei che armonizzino le proposte del progetto europeo (es. il glossario SARMa) è un problema in Ungheria e Romania. La Provincia Autonoma di Trento mette in evidenzia la definizione legale di materiale (naturale, rifiuto o prodotti) che differisce in modo marcato nel processo di produzione: in questo modo vi sono differenti tipi di procedure per lo stesso materiale in funzione della forma legale. Quindi gli aggregati, scavati in una cava, differiscono, da un punto di vista legale, da quelli prodotti in un'opera civile, sebbene entrambi siano geologicamente identici. Ciò coinvolge procedure burocratiche, che sono molto diverse; nonostante gli aggregati abbiano le stesse caratteristiche tecniche e geologiche.

L'estrazione di aggregati da sotto il livello di falda è una problematica sensibile in Austria; in Croazia solo le cave attive sono considerate nei piani territoriali e sono segnate come aree minerarie; in Montenegro vi sono discrepanze fra i piani regolatori dei comuni ed il Piano Nazionale Territoriale. II programma NATURA 2000 limita parzialmente l'attività estrattiva in Austria, Ungheria e Slovenia. Nella Regione EmiliaRomagna il problema principale è la forte gerarchia della pianificazione e il sistema autorizzativo che ha bisogno di tempi lunghi. Nella Bosnia-Erzegovina e in Serbia vi è 
una mancanza normativa sulla pianificazione degli aggregati. La proprietà del suolo causa problemi in Albania e Slovacchia. Mancanza di coordinazione fra le istituzioni coinvolte e responsabili, e piccoli o nessun investimento in ricerche sotterranee e sottomarine di possibili risorse sono i problemi in Slovenia.

\subsection{Conclusioni}

II Quadro di Pianificazione degli Aggregati (basato sulla pianificazione dell'uso del suolo) esiste già in Austria, nella Provincia Autonoma di Trento e Slovenia. In altri Paesi partner i documenti più importanti relativi alla pianificazione degli aggregati sono l'Atto o il Piano Minerario/Estrattivo, la legge sulle concessioni, I'atto Ambientale, la Strategia di sviluppo Sostenibile, l'atto o la Strategia sui Rifiuti e l'atto sulla pianificazione dell'uso del suolo. La Provincia Autonoma di Trento, l'Austria, la Slovenia e l'Albania hanno piani minerali (estrattivi e piani delle risorse minerali, piani strategici minerari); mentre in altri Paesi SEE leggi minerarie (Ungheria, Bosnia-Erzegovina, Croazia, Montenegro), e legge sulla concessione (Bosnia-Erzegovina, Montenegro) si occupano degli aggregati.

Nella maggior parte dei Paesi SEE gli aggregati primari e secondari sono gestiti separatamente es. l'autorità responsabile è differente; o la gestione degli aggregati non è ancora iniziata (Bosnia-Erzegovina, Montenegro, Romania, Serbia, Slovacchia e Turchia). Gli aggregati secondari sono considerati principalmente dalla Politica di Gestione dei Rifiuti dello Stato o regione. La gestione dei rifiuti è in divenire (a diversi livelli) e separata dalla gestione delle risorse. Non tutti i tipi di aggregati secondari sono trattati; per lo più i rifiuti da costruzione e demolizione e in alcuni casi i rifiuti minerari sono menzionati nei documenti legali.

II supporto di SARM (Sustainable Aggregates Resource Management) e SSM (Sustainable Supply Mix) è variabile; in alcuni Paesi sono promossi almeno in teoria (Slovenia, Croazia, Serbia e Montenegro), in altri Paesi non sono noti e non supportati (Ungheria, Bosnia-Erzegovina).

I conflitti potenziali delle attività estrattive e aree ambientali protette sono trattati adeguatamente in tutti i Paesi partner. Questo risulta nella designazione di aree di assoluto divieto per l'estrazione degli aggregati in quasi tutti i Paesi partner (eccetto Romania e Turchia), mentre in base al documento guida sull'estrazione minerale non energetica e Natura 2000 "Non vi è esclusione automatica di attività NEEI (NonEnergy Extractive Industry) all'interno o intorno a Natura 2000. Invece, le attività estrattive dovrebbero seguire le disposizioni delineate nell'articolo 6 della direttiva Habitat per assicurare che queste attività non influenzino sfavorevolmente l'integrità dei siti Natura 2000" (EC 2010a, p. 7,). Le aree di assoluto divieto causano seri problemi in molti Paesi SEE, che potrebbero essere risolti o facilitati dalla consultazione 
con i più importanti portatori di interesse (es. centri decisionali, autorità ambientali, industria estrattiva).

In molti Paesi partner il Quadro Politico di Pianificazione di Uso del Suolo contiene importanti informazioni per la Pianificazione degli Aggregati: es., aree protette naturali o culturali - comunque riguardo le aree designate come minerarie vi è mancanza di informazioni relative agli aggregati. 


\section{Guida sugli Aspetti da considerare nei Piani degli Aggregati}

Lo scopo della Guida è di determinare come rendere migliore la pianificazione nei propri rispettivi Paesi. I partner sono stati invitati a creare un documento-guida che riflettesse la situazione in ogni Paese, e fosse l'input per la rete dei soggetti interessati. Dovevano quindi identificare aspetti di pianificazione che fossero applicabili e rilevanti in molti Paesi SEE poiché il loro inserimento nella Visione Congiunta SNAP-SEE e lo Schema di Pianificazione degli Aggregati aumenterebbe la coesione e l'armonizzazione. 


\subsection{Introduzione}

La politica di pianificazione minerale è parte del quadro nazionale delle politiche minerali (Tiess 2011; EC, 2010). La politica di pianificazione degli aggregati può essere definita come protezione di depositi di aggregati attraverso la pianificazione dell'uso del suolo (ovvero salvaguardando i materiali grezzi). Nel contesto di questo quadro, a livello nazionale una politica di pianificazione degli aggregati deve essere sviluppata considerando le problematiche strategiche che sono poi collegate a livelli di pianificazione regionali/locali (operativi). Ciò è anche un importante principio gerarchico di pianificazione: il processo di pianificazione inizia a (per esempio) 1:100 000 e evolve a scale dettagliate (regionali 1: 25 000; locali $1: 5000$ ).

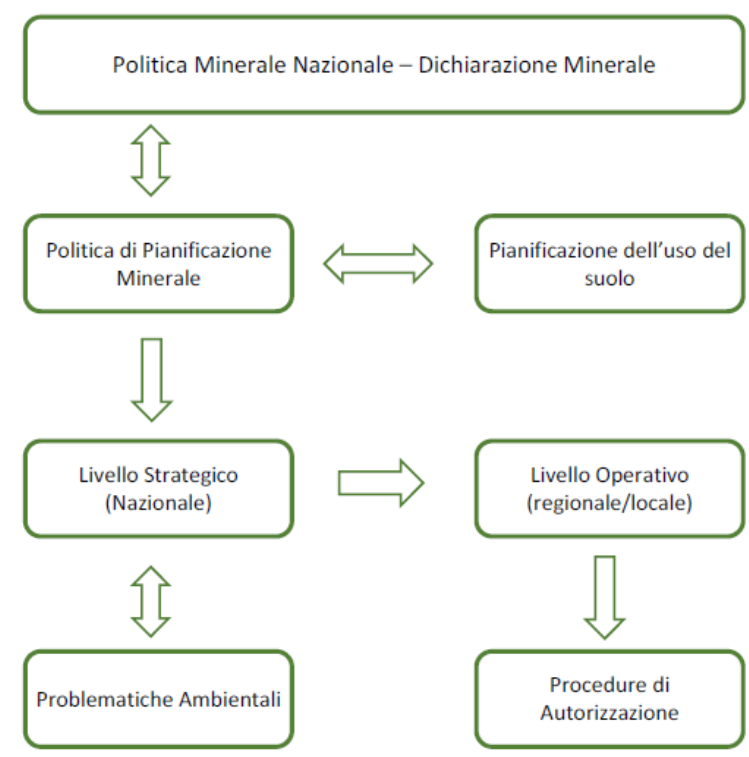

Figura 3.1. Politica Nazionale di Pianificazione Minerale (Tiess 2011; EC 2010b).

"Una politica nazionale dei minerali deve innanzitutto fornire una 'dichiarazione mineraria'. In riferimento agli aggregati due problematiche cruciali si devono comprendere: una politica nazionale mineraria deve prima instaurare la consapevolezza del bisogno di minerali della società, e specificatamente per gli aggregati, ed in questo caso della necessità all'accesso alle risorse locali. La seconda problematica davvero cruciale è che si stabilisce la fornitura di minerali, e in particolare di aggregati, come una risorsa per il beneficio della società, e che si stabilisce un approccio equilibrato nella valutazione dell'esplorazione e sviluppo delle attività estrattive. La politica nazionale dei minerali dovrebbe tener conto della domanda a medio-lungo termine di aggregati, controllando che vi sia una sufficiente scorta di riserve locali con accesso 
che è una caratteristica intrinseca della pianificazione territoriale locale" (Tiess, p. 15, 2010).

La fase di pianificazione regionale è soprattutto importante per la regolamentazione delle problematiche delle materie prime. Con l'aiuto di dichiarazione precise, piani regionali (basati su più ampi programmi di sviluppo, come quelli nazionali e macroregionali) determinano gli obiettivi regionali di uso del suolo per lo sviluppo di singoli distretti. Un piano di uso del suolo regionale designato per l'estrazione e protezione delle risorse minerali deve contenere un diagramma di flusso di pianificazione preciso e una dichiarazione testuale. Quest'ultima inclusa nel piano deve definire "le zone di priorità per gli aggregati" che dovrebbero essere garantite, mentre attentamente si considerano le domande a medio e lungo termine delle risorse minerali, e la disponibilità limitata dei depositi delle risorse minerali.

La determinazione di "zone di priorità per gli aggregati" sconfina nei diritti base di proprietà e quindi richiede un accordo con il proprietario del terreno interessato. Si giustifica se vi è necessità di pubblico interesse, che è vero nel caso di estrazione/fornitura di aggregati, finché gli aggregati sono richiesti per lo sviluppo economico. Tuttavia, la quantità, qualità, e le applicazioni per le quali le risorse saranno necessarie nel medio e lungo periodo dovrebbero essere chiare. Per giustificare la designazione di un'area come "zona di priorità di risorsa per gli aggregati" si richiede un'analisi della struttura del mercato degli aggregati e uno studio del flusso di materiale.

Da un punto di vista metodologico, si possono usare due diversi approcci nella pianificazione territoriale. Il primo è di limitare la pianificazione escludendo certi usi per un'area specifica. Questo metodo ha lo svantaggio, che una fornitura sostenibile di aggregati, ovvero la salvaguardia sistematica delle aree risorse aggregati con priorità, non è possibile. Nel secondo approccio alternativo, i terreni con altre priorità di utilizzo riducono i depositi che vale la pena estrarre mentre i rimanenti terreni diventano aree di priorità della risorsa di aggregati. Il vantaggio di questo approccio è quello che le autorità possono sviluppare una politica concreta della risorsa di aggregati, nonostante il fatto che la speculazione del territorio possa verificarsi. È necessario sviluppare piani di lungo termine che rispondano alla domanda di aggregati, alla disponibilità, e che prevedano gli impatti durante l'intero ciclo di vita dei prodotti (sostenibilità). L'obiettivo finale è di ottenere la sicurezza dell'approvvigionamento e l'efficienza delle risorse.

\subsection{II contenuto dei documenti di pianificazione e i metodi}

Le autorità responsabili per la pianificazione degli aggregati sono autorità minerarie, di pianificazione dell'uso del suolo e altre a livello nazionale e regionale. La loro 
competenza (eccetto la Provincia Autonoma di Trento) copre solo gli aggregati primari.

In 5 Paesi (Albania, Bosnia Erzegovina, Croazia, Slovenia e Provincia Autonoma di Trento) i contenuti della pianificazione aggregati sono predefiniti da un Atto o da un'Ordinanza. I documenti di pianificazione sono la strategia mineraria, l'Atto Minerario e i Regolamenti sulle procedure per concedere le concessioni. In altri Paesi (Ungheria, Romania e Serbia) i contenuti della pianificazione degli aggregati non sono predefiniti da un Atto o da un'Ordinanza, es. in Austria dove il AUT MINPLAN non ha carattere legale.

Nella maggior parte dei Paesi l'autorità principale responsabile (coordinatore) per lo sviluppo e la creazione del piano è il Ministero (dipartimento di politica minerale) - in stretta cooperazione con il servizio geologico nazionale, le autorità di pianificazione dell'uso del suolo e altri ministeri es. dell'Ambiente e della Pianificazione dell'uso del suolo (Ministero della Pianificazione Fisica e pianificazione territoriale).

I piani degli aggregati nella maggioranza dei Paesi sono aggiornati ogni 5 o 10 anni in funzione dalle esigenze della comunità o società, eccetto l'Albania dove la strategia mineraria copre 15 anni e la Bosnia-Erzegovina, Serbia e Romania dove le leggi sul settore minerario non si affrontano in modo specifico la frequenza di aggiornamento dei piani degli aggregati.

In generale un piano degli aggregati dovrebbe basarsi sulla pianificazione dell'uso del suolo. I piani degli aggregati, integrati in piani territoriali, aiutano l'industria e le autorità pertinenti a stabilire un quadro stabile di pianificazione per gli aggregati sul lungo periodo. Dalla sovrapposizione delle mappe di risorse minerali con le aree riservate ad altri scopi di uso del suolo è possibile identificare aree potenziali di conflitto cosicché gli sviluppi futuri possano essere assegnati lontani da queste aree ovunque siano realizzabili.

\subsubsection{Perché dovrebbero le autorità fare pianificazione per l'estrazione minerale?}

La più esauriente riposta per la sopracitata domanda può venire dalla consultazione slovena dei soggetti interessati:

I risultati del workshop interattivo e una discussione hanno suggerito molti modi possibili su come migliorare le pratiche e la legislazione verso una migliore pianificazione dell'approvvigionamento degli aggregati in Slovenia, comprendendo la necessità di una pianificazione territoriale nazionale e di strategie minerarie, miglioramenti del recupero nella legislazione sui rifiuti da costruzione, un suggerimento per un migliore coinvolgimento di diversi soggetti interessati nel processo di pianificazione degli ag- 
gregati, la necessità di meglio distribuire le tasse di concessione fra le comunità locali e lo Stato e l'importanza dell'uso delle migliori tecnologie disponibili per l'estrazione e il trattamento.

\subsubsection{Come dovrebbero le autorità di pianificazione minerale fare pianificazione per l'estrazione minerale?}

Le autorità di pianificazione minerale dovrebbero pianificare per un approvvigionamento regolare e adeguato in uno o più dei seguenti modi (in ordine di priorità):

1. designazione di aree - dove l'attuabile esistenza delle risorse è nota, i proprietari terrieri sono di sostegno allo sviluppo minerario e la proposta è probabile che venga accettata in termini autorizzativi. Tali siti possono quindi comprendere azioni essenziali collegate con l'estrazione minerale;

2. designazione di aree di risorse note dove l'autorizzazione potrebbe ragionevolmente essere anticipata. Tali aree possono quindi comprendere azioni essenziali collegate con l'estrazione minerale;

3. designazione di aree dove la conoscenza della risorse minerale può essere meno certa ma all'interno della quale l'autorizzazione può essere concessa, particolarmente se vi è potenziale scarsità nell'approvvigionamento.

I requisiti SARM del processo di pianificazione/autorizzativo comprendono il fabbisogno per l'adozione di una pianificazione di lungo termine per garantire tempo sufficiente per assicurarsi il ritorno dell'investimento richiesto per iniziare una nuova attività estrattiva. Nei Paesi SEE partner di progetto il piano minerale più completo ottenuto a livello nazionale è il Piano Austriaco delle Risorse Minerali (AMRP) (pubblicato nel 2010). È stato sviluppato in stretta collaborazione con i governi regionali. I piani delle risorse minerali di Paesi come Croazia (Piani Minerali delle Province), Slovenia (Programma Nazionale per la Gestione delle Risorse Minerali - NPMRM) e Provincia Autonoma di Trento, seguono i principi delineati da (Cibin et al, 2011):

1. la domanda di aggregati e la fonte di fornitura: si raccomanda di comprendere una previsione della future domanda di aggregati, basata su dettagliate analisi del mercato degli aggregati e su analisi del flusso di materiali. Se possibile, tali analisi dovrebbero includere l'uso finale previsto dei materiali (tipi differenti di calcestruzzo, materiali da riempimento, basi stradali, superfici stradali, ecc.)

2. disponibilità di aggregati: il piano di approvvigionamento dovrebbe comprendere la posizione degli aggregati naturali ed alternativi. Questi dovrebbero comprendere tutte le possibili alternative fonti, e le risorse dovrebbero quindi venire descritte in termini della loro qualità. Si raccomanda di valutare l'atteso esaurimento di risorse non rinnovabili che dovranno venire estratte 
per soddisfare la domanda prevista. Lo scopo è di ridurre questo valore al minimo.

3. impatti potenziali: la quantificazione di tutti gli impatti locali è necessaria (rumore, aria, risorse idriche, biodiversità, ecc.); un'analisi dei principali percorsi che saranno usati per il trasporto della fornitura dovrebbero essere esaminati, comprendendo gli impatti attesi su questi percorsi.

4. Analisi del Ciclo di Vita: la destinazione degli aggregati (recupero/smaltimento) al termine del loro ciclo di vita è importante, come sono le modalità di ripristino di tutte le aree esauste di cava.

5. Scenari (fornitura/domanda): approcci alternativi al soddisfacimento della domanda di aggregati necessitano d'esser considerati.

Come scritto nell'introduzione, i depositi di materiali da costruzione, cioè aggregati, hanno tre caratteristiche principali: la loro posizione determinata per natura, l'impiego locale e l'esauribilità. Per via di queste proprietà l'esistenza di depositi, come anche la loro estensione e costituzione necessitano di essere determinate prima di altri usi possibili. L'accessibilità al deposito in un secondo momento dipende dalla pianificazione dell'uso del suolo in considerazione di tutti gli aspetti rilevanti. Informazioni aggiornate e complete sui depositi aiutano a migliorare la soluzione di potenziali conflitti di uso nella pianificazione d'uso del suolo. Quindi il punto precedente comprende la conoscenza base necessaria per sviluppare un piano di fornitura degli aggregati. Ottenere la raccolta di questi dati al più elevato (strategico) livello (regionale a nazionale) è più efficiente e desiderabile, e per poi fornirlo al livello locale (alle autorità competenti). Questo sarà cruciale per il monitoraggio dello sviluppo dei mercati degli aggregati nel medio-lungo termine, e per la riduzione della diversità nei piani fra le regioni adiacenti (Cibin et al, 2011).

Esempio : Contenuto di un Piano Minerale Regionale (Croazia)

1. Mappe di risorsa minerale potenziale geologica (scala da 1: 10000 a 1: 100 000)

2. Banche dati sui siti di risorsa minerale

3. Aree di sfruttamento

4. Dati di produzione e riserve

5. Condizione di protezione ambientale e restrizioni

6. Fabbisogno del mercato e dello sviluppo

7. Raccomandazioni per i ripristini

8. Aree delineate più favorevoli per siti estrattivi (aree con beni minerali non in conflitto con altri usi del suolo)

9. Opinioni dei portatori d'interesse sulle politiche minerali (autorità locali, operatori, NGOs, funzionari comunali) 
Esempio: Il piano austriaco delle risorse minerali

Il Piano Austriaco delle Risorse Minerali (AMRP) indica le zone di estrazione degli aggregati che sono/dovrebbero essere importanti per il processo di pianificazione regionale: ogni legge di uso del suolo deve considerare le cosiddette zone di priorità degli aggregati che dovrebbero essere mantenute libere da altre ipotesi di sviluppo/utilizzo. Tali zone sono indicate sul AMRP e verrebbero comprese nei piani di uso del suolo regionali (processo in corso). Come pianificazione regionale devono essere recepite le aree di priorità degli aggregati - assicurate da piani regionali - e devono essere accettate dai governi locali (gerarchia di pianificazione). Se un operatore vuole estrarre aggregati, deve fare riferimento a quelle zone.

(Si veda quindi l'esempio nel capitolo 3.2.3)

\subsubsection{Geologia e potenziale di aggregati}

Nei documenti ufficiali di pianificazione dei Paesi SEE la mappa geologica (potenziale) esiste in varie scale partendo da scale di 1:500 000 a livello nazionale (Ungheria Piano Nazionale di Uso del Suolo, Romania), ma anche dettagli da 1:50 000 per le aree più interessanti (Romania, Ungheria - una mappa delle aree di gestione delle risorse minerali è parte del Piano Nazionale di Uso del Suolo, e i siti minerari sono segnati in province e piani locali di uso del suolo), fino a Trento con le mappe provinciali geologiche - scala 1:10 000. La mappa geologica albanese esiste in varie scale partendo da 1:200 000, 1:50 000 e 1:25 000 per quasi il 75\% del territorio sono realizzate mappe geologiche a scale 1:10 000. In Austria i dati per l'intero Paese sono disponibili a scala 1:50 000. In Croazia esistono dati geologici limitati; nei piani di uso del suolo a scala 1:100 000 (Piano Regionale di Uso del Suolo). In Slovenia tutti i dati geologici all'interno del lavoro del Servizio Pubblico Minerario, GeoZS, che provvede alle revisioni, pareri e guide (che sono basate sui dati geologici disponibili, comprendendo le banche dati e le mappe) per la pianificazione territoriale/urbanistica alle autorità. In Serbia esistono dati geologici di base e alcune mappe minerali generali ma non sono comprese nei piani territoriali/urbanistici. Nella maggioranza dei Paesi i dati sono disponibili in sistemi GIS che possono assicurare nel futuro calcoli, calibrazioni e sottili aggiustamenti che potrebbero essere fatti per la maggior parte automaticamente e che la veridicità dei risultati potrebbe essere verificata in ogni momento.

I minerali possono essere estratti solo dove esistono naturalmente, cosicché le opzioni di posizione per un'estrazione sostenibile da un punto di vista economico e accettabile da quello ambientale può essere limitata. Questo significa che è necessario considerare tutte le informazioni geologiche (mappe, rapporti, ecc.) sulla presenza di aggregati sia a scala nazionale che regionale. I principi delineati nel Piano Austriaco delle Risorse Minerali (AMRP) sono un esempio che dovrebbe essere seguito quando si prepara un quadro geologico per un documento di pianificazione degli aggregati. In 
geologia, come nella pianificazione territoriale, le valutazioni sono basate su scale di mappe che possono essere differenti in ogni Paese principalmente in funzione dei Programmi di Mappatura Geologica. Nei Paesi SEE le scale variano da 1: 10000 a 1:200 000. Le scale regionali privilegiate variano fra 1:25 000 a 1:50 000.

\section{Esempio: Mappe di sabbia e ghiaia}

La valutazione della presenza di sabbia e ghiaia in Austria (AMRP) è basata su mappe ad una scala di 1:50 000 descriventi Paleogene non consolidato, Neogene e sedimenti del Quaternario. Questa mappa mostra sia la mappa regionale dalle facies di sedimenti non consolidati, sia informazioni in merito alle caratteristiche litologiche più importanti del materiale. Un approccio simile è anche compiuto in Croazia dove i dati sono disponibili a scala 1:100 000 .

La valutazione della presenza di sabbia e ghiaia si basa sulla qualità e quantità del materiale grezzo e sull'importanza dell'aree di approvvigionamenti locali o regionali. Questi tre criteri sono stati combinati in una matrice 3D per dedurre l'idoneità geologica della sabbia e ghiaia in un Sistema Informatico Geografico (GIS). La valutazione della qualità dei materiali grezzi si basa su due fattori: (1) la descrizione litologica del materiale e (2) l'uso del materiale grezzo.

Per calcolare il potenziale geologico di un deposito di sedimento, la qualità e quantità del materiale vengono combinate in una prima matrice. Differenti matrici sono state usate per calcolare il potenziale nei Foreland e in aree montane cosicché specifiche caratteristiche geologiche regionali potevano venire prese in considerazione, così come le piccole presenze in valli alpine che possono essere importanti per la fornitura locale così come i grandi depositi in aree vicino alle città più grandi. Le risultanti fasi di quantificazione del potenziale descrivono la capacità relativa dei corpi di sedimenti per fornire sabbia e ghiaia come materiale da costruzione.

Esempio: Mappe di roccia frantumata/massi naturali

La classificazione geologica della presenza e dei depositi è stata condotta sulla base della mappe geologiche austriache a scala 1:200 000 e, dove disponibili, 1:50 000. La fonte dell'indagine dei siti minerari è stato l'archivio del Servizio Geologico che è regolarmente aggiornata sulle cave e i dati dei siti minerari abbinato al catalogo digitale e al sistema di ricerca.

Nell'industria edile e dell'ingegneria civile si comprendono i prodotti frantumati (roccia frantumata, brecciame anche di alta qualità, sabbia frantumata) come anche pietre da taglio. Le rocce solide che vengono frantumate sono (1) rocce magmatiche, (2) metamorfiche e (3) sedimentarie. Per ognuno di questi materiali grezzi, vi indicata la presenza o l'esplorazione (aree di materiali grezzi nel senso ampio) o le modalità d'estrazione (di solito cave, raramente miniere sotterranee) 


\subsubsection{Inventario Minerali/ Aggregati}

Nella maggioranza dei Paesi SEE esiste un inventario minerali/aggregati, eccetto in Bosnia Erzegovina e Serbia. In altri Paesi SEE l'inventario esiste e include tutti i dati per le aree minerarie con le coordinate GIS, posizione, dimensioni dell'area, tipo di minerale, genere di attività (esplorazione o sfruttamento), tipo di riserva e quantità di riserva, dati qualitativi sui minerali come la percentuale di minerale di valore.

I dati delle risorse, le riserve di aggregati primari nonché i dati della produzione, dovrebbero idealmente consistere come una parte del GIS dei minerali di:

1. dati territoriali relativi alle dimensioni e forma del deposito sfruttabile;

2. riserve comprovate;

3. tipi e qualità di aggregati.

I dati disponibili nella maggior parte dei Paesi sono limitati a causa della riservatezza ma sono di solito raccolti su base annuale dalle autorità minerarie.

\subsubsection{Inventario degli aggregati secondari}

Nella maggioranza dei Paesi SEE partner di progetto gli aggregati secondari hanno inventari poveri. In queste nazioni il Ministero o l'Agenzia per l'Ambiente forniscono $i$ dati sui rifiuti (C\&D, minerari, aggregati secondari) in accordo con la direttiva Rifiuti o leggi similari. Quattro partner SEE non hanno una banca dati dell'inventario degli aggregati secondari o hanno alcuni dati parziali (Bosnia Erzegovina, Serbia, Albania e Romania). 
Esempio: Provincia Autonoma di Trento

Il piano stralcio rifiuti da C\&D ha I seguenti contenuti:

1. Un rapporto tecnico contenente:
a. Tipi e quantità di rifiuti da gestire;
b. Metodi di trattamento;
c. Dimensione dell'utilizzatore base;
d. Criteri di organizzazione e gestione;

2. Localizzazione degli impianti di smaltimento e recupero e individuazione delle aree adeguate, dove tali impianti possono essere localizzati;

3. Individuazione di aree adeguate per la localizzazione degli impianti di smaltimento e recupero in conformità con le direttive e previsioni del piano urbanistico provinciale e di altri piani sovraordinati;

4. I criteri e le norme tecniche per la progettazione, installazione e gestione degli impianti;

5. L'identificazione dell'accesso agli impianti;

6. Grafici in scala adeguata per evidenziare i contenuti del piano.

Il Servizio minerario della Provincia è responsabile per il piano minerario che lo redige. L'Agenzia per l'ambiente provinciale è responsabile per il piano stralcio rifiuti da C\&D e lo redige. In entrambi i casi altri servizi pubblici e associazioni di soggetti interessati sono coinvolti nella stesura del piano: es. il servizio geologico e l'associazione delle imprese estrattive. Organizzazioni esterne, come studi professionali, possono essere coinvolti nella redazione del piano: es. il piano stralcio rifiuti da $C \& D$ è stato redatto con la collaborazione di un professionista esterno. I piani sono approvati dalla Giunta provinciale. 
Esempio: Metodologia per tracciare gli aggregati riciclati (Ontario, Canada)

Basandosi sui risultati di un'indagine delle agenzie pubbliche e un esame di sistemi internazionale simili, quello che segue è il metodo raccomandato per tracciare gli aggregati riciclati in Ontario:

1. Si dovrebbero sviluppare linee guida su come e quali materiali da tracciare per standardizzare i dati da inserire nel sistema da tutta la provincia.

2. Una banca dati computerizzata online dovrebbe essere sviluppata per fornire un mezzo per le agenzie pubbliche per registrare le loro informazioni riguardo l'uso degli aggregati riciclati.

3. Affinché le agenzie pubbliche siano in grado di compiere questa mansione supplementare, richiederanno maggiori investimenti per lo staff e formazione.

4. Per considerare le agenzie pubbliche responsabili di questa mansione supplementare, gli investimenti integrativi dovrebbero essere legati alla registrazione propria dei dati.

5. Per promuovere i benefici sociali del recupero, pagelle annuali dovrebbero essere create evidenziando i successi e le opportunità per il miglioramento nell'uso degli aggregati riciclati all'interno della provincia.

Fonte: Ministero delle Risorse Naturali dell'Ontario (2010)

\subsubsection{Economia degli Aggregati}

La maggior parte dei Paesi SEE partner di progetto non raccolgono e non hanno dati statistici o modelli o previsioni in merito all'andamento del consumo degli aggregati e di produzione. Solo la Provincia Autonoma di Trento ha dati riguardo i volumi di costruzione, ma un modello non è attualmente implementato.

La valutazione dell'economia degli aggregati dovrebbe fare molto affidamento sul concetto di Mix di Approvvigionamento Sostenibile (SSM - Sustainable Supply Mix). Le fonti multiple degli usi del SSM che insieme massimizzano il beneficio netto della fornitura di aggregati nel tempo (Shields et al., 2006). La pianificazione SSM è collegata al processo di pianificazione/sviluppo dai rispettivi portatori d'interesse/autorità usando queste fonti multiple per assicurare un approvvigionamento sostenibile degli aggregati. Tutti i soggetti interessati, es., quelli responsabili per la pianificazione dell'uso del suolo o per la regolamentazione del processo di pianificazione degli aggregati riciclati e l'uso sostenibile delle risorse naturali, dovrebbero agire in stretta cooperazione. II quadro pianificatorio SSM fornisce "regole operative di base" per l'industria degli aggregati primari e secondari. L'industria degli aggregati stessa è responsabile per l'intero ciclo di vita della cava comprendendo esplorazione, sfruttamento, trattamento e ripristino dei siti, e dovrebbero organizzare di conseguenza i 
loro affari. Il problema si erge (problematiche sociali) a quale "grado" gli operatori e i comuni interessati dovrebbero essere coinvolti nel processo di pianificazione SSM. Per esempio, gli operatori spesso non sono soddisfatti con le zone selezionate di priorità degli aggregati in termini di priorità tecniche con riguardo ai prezzi dei terreni.

\section{Esempio: Mercato minerale degli aggregati}

II mercato minerale degli aggregati degli Stati Uniti dell'Est è modellato quantitativamente e l'interazione dei suoi componenti sono misurate. Lo studio sistematico delle caratteristiche di fornitura, domanda, prezzo e trasporto degli aggregati ha individuato parametri di modellazione importanti. II modello di fornitura è basato sui dati di produzione. La domanda stima il consumo totale sulla base del prodotto pro capite e dei tassi d'interesse. Il prezzo dipende principalmente dalla disponibilità geologica e dalla dimensione del mercato, dalla dimensione dell'operazione e dal reddito pro capite medio annuale. La consegna è il modo di minimizzare i costi equilibrando produzione e consumo, dati i vincoli imposti dai costi di trasporto.

Sebbene i prezzi varino da Paese a Paese, gli aggregati sono relativamente prodotti a basso costo. I margini di profitto sono di solito molto stretti e il settore aggregati è spesso molto sensibile a qualsiasi fattore esterno diretto o indiretto che possa influenzare i prezzi. Gli aggregati sono molto ingombranti e sono di solito consumati da mercati vicini al punto di estrazione. Il trasporto è un elemento chiave del processo di fornitura e una gran parte del prezzo alla consegna. Il costo di un carico camionabile di aggregati raddoppia nel movimentarlo di $40 \mathrm{~km}$. I concetti di approvvigionamento e gestione degli aggregati dovrebbero basarsi su analisi dettagliate del mercato degli aggregati, studi sui flussi di materiale e simulazioni di trend. In questo senso, è importante distinguere fra consumo e produzione, così come fra fornitura interna (locale/regionale) ed esterna (interregionale, trans regionale). Un quadro di politiche SSM dovrebbe provvedere all'industria degli aggregati il supporto necessario, es. l'accesso al territorio, e altre informazioni pertinenti come la logistica per il trasporto.

Fonte: Poulin, R., Bilodeau, M.L. (1993)

\subsubsection{Pianificazione territoriale/urbanistica e impatti ambientali}

La maggior parte dei partner SEE hanno alcune procedure e criteri per l'identificazione delle zone di sfruttamento. Alcuni Paesi hanno certe condizioni pianificatorie molto ben definite. Il quadro di pianificazione dell'uso del suolo in Austria è molto complesso e complicato; mentre nella Provincia Autonoma di Trento vi sono due strumenti per la valutazione: il comitato interdisciplinare e la VIA. La VIA è obbligatoria nella maggior parte dei Paesi per tutte le attività minerarie e altri interventi in 
merito all'ambiente. In alcuni Paesi non vi è sufficiente cooperazione fra autorità nella determinazione dei criteri ambientali, zone di buffer e trasporto.

Le problematiche principali che sono relative alle autorità di pianificazione degli aggregati dovrebbero trovarsi nei documenti di pianificazione, tenendo presente che non tutte le problematiche sono rilevanti per ogni sito ed allo stesso grado:

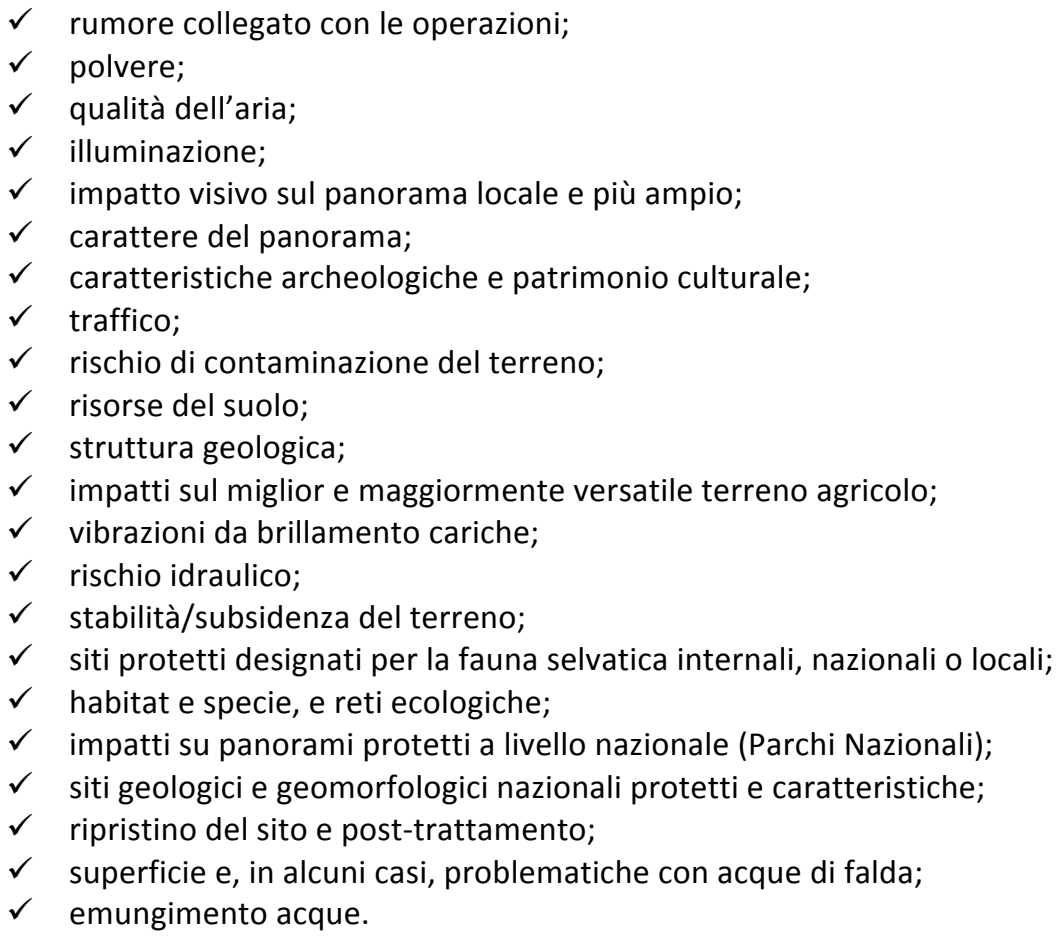

\section{Esempio: Problematiche di trasporto e impatti ambientali}

Nella Fase 2 del Piano Austriaco delle Risorse Minerali, le aree di materiale grezzo identificate e mappate nella Fase 1 sono state unite digitalmente con quelle dei piani di sviluppo regionale che proibiscono o impediscono l'estrazione dei materiali grezzi (eliminazione dei conflitti). Nel caso di superfici vicino ai materiali grezzi, le aree sono state scelte sulla base della necessità. Uno sforzo è stato compiuto per minimizzare la distanza dal produttore al consumatore. Ridurre le distanze di trasporto mediamente del $10 \%$ poteva tagliare le emissioni annuali di $\mathrm{CO}_{2}$ di più di 1 milione di tonnellate. II trasporto causa vibrazioni, rumore e polvere che spesso non si possono evitare. Gli operatori tentano di trovare percorsi adeguati per minimizzare il numero di abitazioni colpite. Ciò spesso comporta in un aumento delle distanze di trasporto. 


\subsubsection{Procedura metodologica - estrazione di materiale grezzo e pianificazione}

dell'uso del suolo

La ricerca Joanneum ha sviluppato una metodologia efficace per la valutazione dei conflitti che potrebbero avvenire nell'estrazione minerale previsti all'interno del Piano Austriaco delle Risorse Minerali (Figura 3.2). Il fulcro di questa procedura è I'utilizzo di varie mappe tematiche, cosiddette mappe naturali potenziali, dove certe informazioni riguardo all'estrazione dei materiali grezzi sono state a posta selezionate e sovrapposte. La sovrapposizione tematica di differenti tipi di utilizzo individua certi conflitti, che sono classificati secondo la loro priorità e di seguito risolti.

La procedura di valutazione include i seguenti passi:

$\checkmark \quad$ Tutti gli utilizzi rilevanti

$\checkmark \quad$ La situazione idrologica

$\checkmark \quad$ I depositi minerali superficiali

È stato quindi ottenuto un insieme di mappe tematiche contenenti informazioni significative e parametri per la pianificazione. Basandosi su queste configurazioni, sono state individuate le cosiddette "aree positive e negative" riguardanti l'estrazione dei materiali grezzi e costituiscono le fondamenta per i centri decisionali. Il concetto e il metodo utilizzati nella preparazione delle mappe sono retti da due obiettivi: primo, sviluppare un sistema per i pianificatori che sia trasparente e flessibile per tener conto di input e nuove informazioni e, quindi, identificare nuovi prodotti; secondo, fornire una base per altri usi pianificatori nell'ottica di rendere il sistema di mappe il più versatile possibile. 


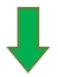

Selezione di aree di divieto di sfruttamento secondo la Legge Mineraria

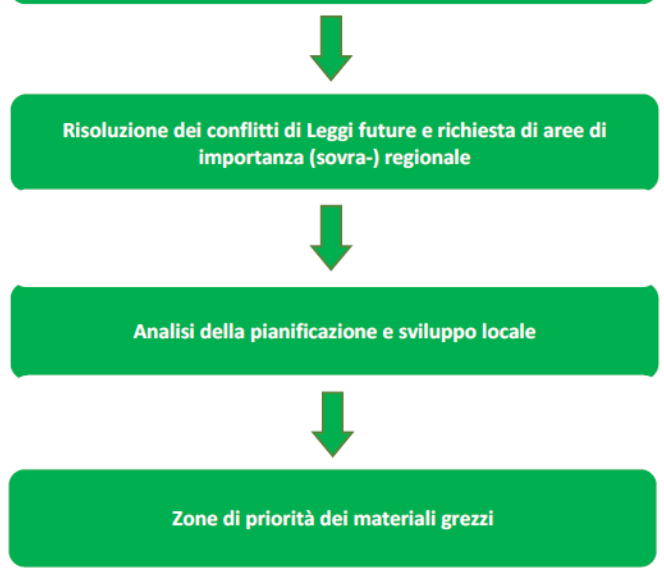

Figura 3.2. Metodo di Pianificazione Applicato all'interno del quadro del Piano Austriaco delle Risorse Minerali (ovvero i livelli di pianificazione nazionale e regionale)

Le mappe delle zone d'idoneità redatte usando metodi geo-scientifici sono sovrapposte digitalmente ai dati di pianificazione resi disponibili dai governi provinciali. Le rispettive specificazioni regionali di pianificazione sono confluite nelle cosiddette zone di divieto o di conflitto. Zone di divieto sono quelle aree nelle quali l'estrazione dei materiali minerali grezzi è proibita da leggi federali o provinciali. Zone di conflitto sono definite come quelle aree in cui vi sono impedimenti all'estrazione. Queste aree includono, per esempio, aree tipo Natura 2000, dove i materiali grezzi possono solo essere estratti se vi è un'analisi positiva di compatibilità naturale. Le singole province definiscono le zone di conflitto in modo molto diverso, sia nei contenuti che nell'ambito.

I risultati della sovrapposizione delle mappe delle zone d'idoneità con le zone di divieto sono state riferite allo Scenario 1 . Successivamente, le aree di conflitto sono state ritagliate e le aree rimanenti (rappresentazione residuale) sono confluite nello Scenario 1. Queste aree residuali sono in sostanza aree di materiali grezzi dove i conflitti sono stati eliminati, ma che richiedono comunque un'analisi attenta, in particolare le aree residuali piuttosto piccole per permettere un'estrazione fattibile econo- 
micamente o altre in cui vi sono ragioni che limitano future estrazioni (es. parchi eolici, linee elettriche, gasdotti ecc.).

\subsubsection{Distanze/zone buffer di separazione}

Distanze di separazione/zone di buffer possono essere appropriate in specifiche circostanze dove è chiaro che, basandosi su precise valutazioni in situ e altre forme di misure di mitigazione (come progettazione di schemi lavorativi e architettura del paesaggio) una certa distanza è richiesta fra i limiti dell'area estrattiva e la zona residenziale abitata. Alcune distanze di separazione proposte si dovrebbero stabilire su una base specifica in sito e dovrebbero essere efficaci, giustificate propriamente, e ragionevoli. Si dovrebbe tenere in considerazione:

$\checkmark \quad$ La natura dell'attività estrattiva minerale;

$\checkmark \quad$ La necessità di evitare una non necessaria inibizione delle risorse minerali;

$\checkmark$ Posizione e topografia;

$\checkmark \quad$ Le caratteristiche di vari effetti ambientali che probabilmente si verificheranno;

$\checkmark \quad$ Varie misure di mitigazione che possono essere messe in pratica.

Una volta che le aree residue da entrambi i scenari sono state consolidate in questo raffinato processo di messa a punto, viene condotta un'analisi volumetrica.

AMRP - come citato sopra - è interrelato con i piani regionali di uso del suolo. I piani regionali sono basati sulle leggi di pianificazione di uso del suolo e sui programmi di sviluppo (livello regionale) e si devono rispecchiare nei piani locali di uso del suolo. Un piano regionale di uso del suolo designato per l'estrazione e protezione delle risorse minerali deve contenere una precisa raffigurazione della pianificazione e una dichiarazione testuale. La dichiarazione testuale inclusa nel piano deve definire il termine "zone di priorità di materiale grezzo" cosicché queste aree servano allo scopo di estrazione nelle parti dell'area sotto pianificazione, in cui l'estrazione dei minerali dovrebbe essere garantita, mentre attentamente si considerano le domande di risorsa minerale a medio e lungo termine, e la disponibilità limitata dei depositi di risorsa minerale (rispettivamente questioni di ripristino).

\subsubsection{Analisi di zone di priorità degli aggregati}

L'analisi delle zone di priorità degli aggregati si basano su:

1. Esclusione di aree con divieto d'estrazione secondo le direttive della Legge Mineraria (Articolo 82): area di sviluppo abitativo (compresa 
l'area del centro) e $300 \mathrm{~m}$ di spazio libero, altri terreni costruttivi, aree di protezione idrica e aree protette;

2. Risoluzione di conflitti con rilevanti pretese di superficie come zone di priorità agricola, zone verdi e restrizioni collegate a altre leggi (corpi idrici e aree forestali);

3. Risoluzione di conflitti con la pianificazione locale d'uso del suolo, es. pianificazione di progetti stradali.

\subsubsection{Aspetti Sociali}

La maggioranza dei Paesi SEE partner di progetto hanno obbligatoriamente incontri pubblici durante il processo d'autorizzazione, in questo modo i soggetti interessati sono coinvolti nella pianificazione territoriale/urbanistica. In alcuni Paesi gli aspetti sociali sono trattati dall'opinione di esperti nel rapporto Geologico e nei piani Minerari e in documenti (Serbia, Romania e Slovenia). Ma la produzione di aggregati non è valutata attraverso metodi di analisi multi criteri basati sulla classificazione e la preferenza dei vari soggetti interessati.

\subsubsection{Ripristini, e uso vantaggioso successivo}

Nella maggior parte dei Paesi SEE partner di SNAP le società minerarie hanno un requisito obbligatorio per ottenere l'autorizzazione di scavo, cioè di elaborare un piano di ripristino, così è scritto nell'Atto Minerario, nel Piano Generale del Programma Nazionale per la Gestione delle Risorse Minerali che contiene le linee guida generali per i ripristini o nel Programma di Sviluppo Regionale. I problemi sono i siti estrattivi inattivi (abbandonati). Nella Provincia Autonoma di Trento cave abbandonate senza ripristino non rispecchiano la pratica legale, ma in quel caso il Comune può rivalersi sul deposito precauzionale (cauzione) per il ripristino: infatti la società estrattrice deve accollarsi i costi di messa in pristino e, se la società fosse inadempiente, perderà la cauzione a favore del Comune.

L'autorità mineraria (ispettorati) controlla se la produzione è conforme a come l'imprenditore ha dichiarato. L'autorità mineraria monitora a campione cave selezionate o basandosi su denuncie. La responsabilità per il ripristino e il post-trattamento di un sito estrattivo, comprendendo la responsabilità finanziaria, spetta all'operatore e, nel caso di bancarotta, al proprietario del terreno.

La forma più appropriata di ripristino di un sito per facilitare differenti usi successivi potenziali dovrebbe essere affrontata nei piani minerali locali , che dovrebbero includere politiche per assicurare che il terreno lavorato sia ripristinato alla primissima opportunità e che un ripristino d'alta qualità e post-trattamento del sito abbiano 
luogo, e abbiano luogo conseguenti confronti sito per sito fra operatori e autorità di pianificazione.

II livello di dettaglio richiesto sul ripristino e post-trattamento dipenderà dalle circostanze di ogni specifico sito comprendendo la durata attesa delle operazioni nel sito. Deve essere sufficiente dimostrare chiaramente che gli obiettivi globali della programmazione sono praticamente raggiungibili e normalmente includerebbero:

$\checkmark \quad$ Una strategia complessiva di ripristino, identificando gli usi successivi proposti del sito;

$\checkmark \quad$ Informazioni riguardanti le risorse del suolo e l'idrologia, e come il terreno superficiale/sottosuolo/terreno di copertura/suolo sia da trattare finché l'estrazione è in funzione;

$\checkmark$ Dove il terreno è agricolo, una valutazione del grado di classificazione del terreno agricolo;

$\checkmark$ Strategia paesaggistica.

Dove lo scavo è proposto nei terreni agricoli migliori e più versatili, la strategia di contorno dovrebbe mostrare, dove praticabile, come i metodi utilizzati nel ripristino e post-trattamento permettano al terreno di conservare la sua capacità di lungo periodo, sebbene la necessità del proposto uso successivo non sia sempre agricolo.

Vi sono molti usi del terreno possibili una volta che l'estrazione minerale è completa e il ripristino e il post-trattamento del terreno sono completi. Questi includono:

$\checkmark$ Creazione di nuovi habitat e biodiversità;

$\checkmark$ Uso per agricoltura;

$\checkmark$ Selvicoltura;

$\checkmark$ Attività ricreative;

$\checkmark$ Gestione dei rifiuti, incluso deposito rifiuti;

$\checkmark$ Ambiente costruito, come residenziale, industriale e commerciale dove appropriato. 


\section{Visione congiunta sui contenuti ottimali dei Piani degli aggregati}

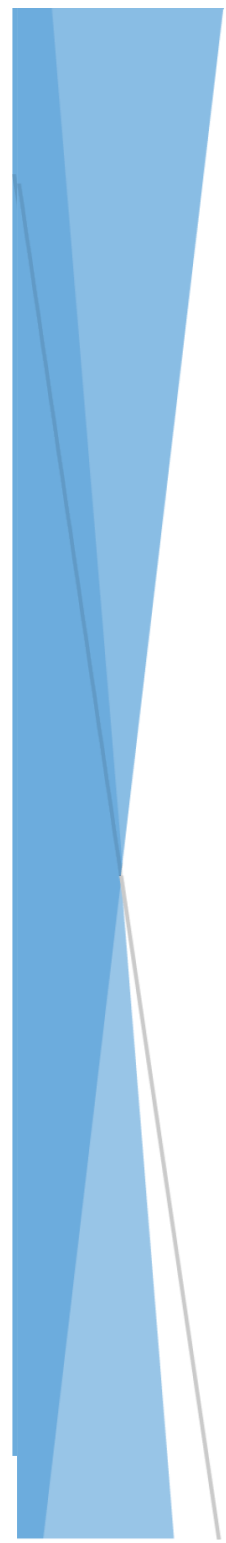

Assicurare un accesso affidabile e semplice ai materiali grezzi è un fattore di crescente importanza per la competitività economica della UE. Uno dei pilastri dell'Iniziativa sui Materiali Grezzi (adottata dalla Commissione Europea nel novembre 2008) è di stabilire le condizioni per un corretto quadro normativo all'interno dell'Unione per favorire una fornitura sostenibile da fonti europee (Guida CE 2010).

I più importanti aspetti della pianificazione degli aggregati (al di fuori della regione SEE: es. la pianificazione britannica, all'interno della regione SEE: es. l'Austria) sono da bilanciare e avere politiche sostenibili sulle risorse minerali. Due cose hanno bisogno di essere coordinate. Primo le condizioni della società che desideriamo e secondo che il suo benessere non sia compromesso.

Questa è una sfida importante per i 13 Paesi SEE e per questi scopi l'uso più efficiente di materie prime seconde è essenziale. Questa visione congiunta è necessaria per lo sviluppo di strategie nazionali, transnazionali e macroregionali europee che possono essere gestite bene. 


\subsection{Introduzione}

La visione congiunta è stata fatta basandosi sui risultati dell'analisi multisettoriale, la guida della pianificazione degli aggregati, le esperienze delle consultazioni dei soggetti interessati, I'analisi SWOT (principalmente secondo il Servizio Geologico Britannico), raccomandazioni SARMa (Sustainable Aggregates Management - Gestione Sostenibile degli Aggregati) e su altri aspetti (es. SIP EIP RM 2013)(Figura 4.1).

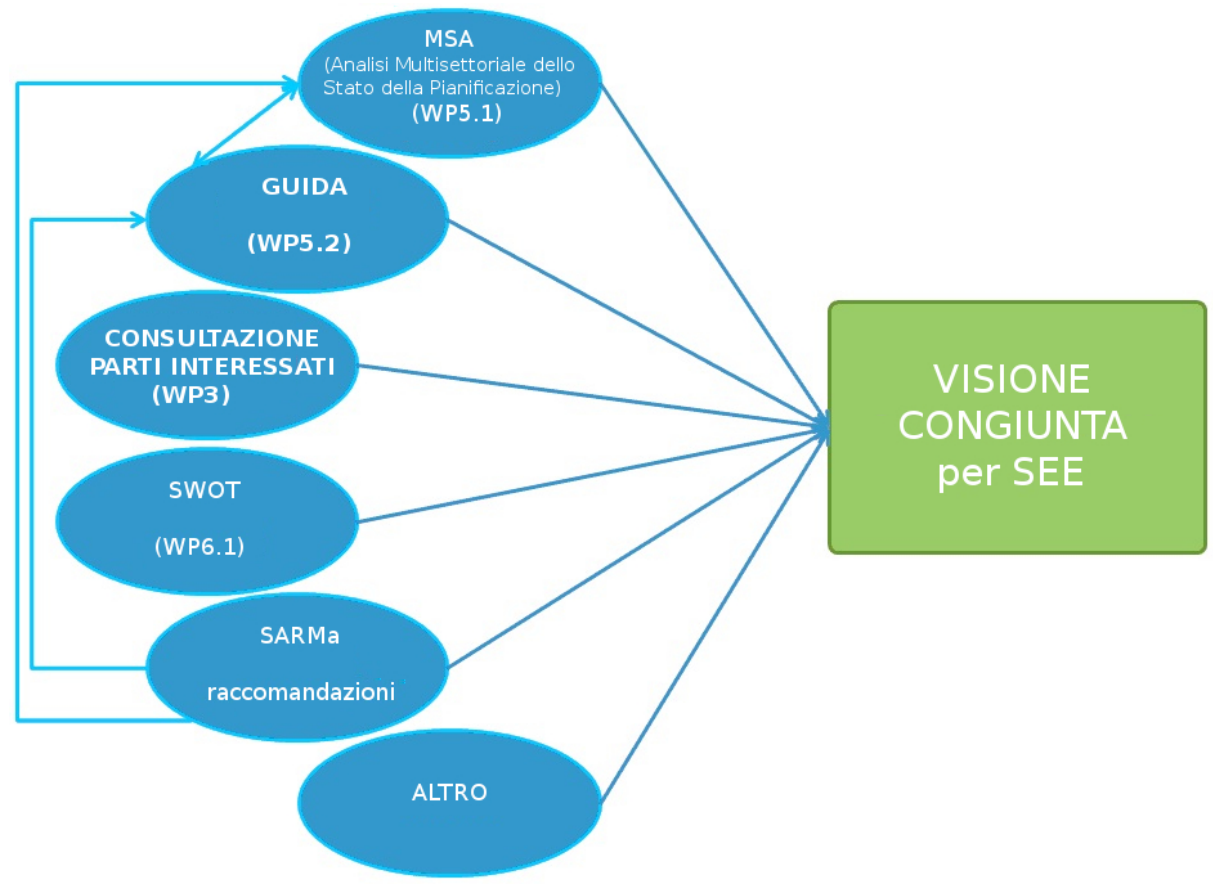

Figura 4.1. Fonte dei dati per la Visione Congiunta SEE

La visione congiunta per la pianificazione degli aggregati nei Paesi del Sud Est Europa è organizzata dai seguenti argomenti: quadro legale-normativo (livello nazionale, pianificazione degli aggregati, altre politiche collegate, procedure di autorizzazione, coinvolgimento dei soggetti interessati, pianificazione locale, autorità, problematiche EU), problematiche ambientali (inquinamento dell'aria, rumore, delle acque, sanzioni minerarie e rifiuti, compensazione ecologica), problematiche sociali (aumentata conoscenza e consapevolezza), problematiche economiche (politiche SARM transnazionali, tassazione), incarichi professionali (inventario, salvaguardia minerale), tecnologia (riciclaggio, brillamento, ripristino, sicurezza), generale (trasporto).

II testo è scritto nei periodi indicanti che fiduciosamente dichiarano che le sfide saranno quasi del tutto svolte in tutti i Paesi SEE per il 2020. Dopo questo periodo lo 
stato della pianificazione sostenibile degli aggregati dipenderà dai cambiamenti nelle legislazioni nazionali ed europee.

\subsection{Quadro legale normativo}

\subsubsection{Livello Nazionale}

\section{Pianificazione degli aggregati}

La Pianificazione degli Aggregati è compiuta a livello statale, regionale e locale, e questi livelli sono armonizzati. La pianificazione nazionale è un approccio generale, mentre i piani regionale e locali sono più dettagliati e hanno una risoluzione più elevata. La Politica di Pianificazione degli Aggregati è basata sulla Politica di Pianificazione di Uso del Suolo integrando altre politiche (pianificazione minerale, sviluppo sostenibile, gestione ambientale e dei rifiuti e politiche di recupero/riciclaggio). Nella pianificazione degli aggregati gli aggregati primari e secondari sono gestiti insieme per proteggere le risorse di aggregati primari e prevenire lo smaltimento dei rifiuti. II contributo dei materiali secondari è tenuto in considerazione nei piani locali prima di considerare l'estrazione di materiali primari. La pianificazione è gestita da autorità pianificatrici in cooperazione con diversi settori (autorità pianificatrici, autorità geologiche e minerarie, industria degli aggregati, dipartimenti governativi, organizzazioni nazionali ambientali e del patrimonio culturale). Durante lo sviluppo della Politica di Pianificazione degli Aggregati, i soggetti interessati sono coinvolti, cosicché le Politiche passino attraverso una procedura di consultazione pubblica. Per questo sempre più difficile accesso alla risorsa, i Piani degli Aggregati devono guardare almeno 20 anni in avanti ed essere regolarmente aggiornati, es. ogni 5-10 anni, in funzione delle esigenze della comunità o società. La previsione di domanda è proiettata nel futuro di 10-15 anni e rivista annualmente, è basata su una media mobile di 10 anni di dati di vendite e altre informazione pertinenti, e su una valutazione di tutte le opzioni di fornitura (includendo le fonti secondarie). II piano degli aggregati è sviluppato per soddisfare questi fabbisogni. Le riserve autorizzate soddisfano i fabbisogni per almeno 5-10 anni. La pianificazione degli aggregati tiene conto dell'ottimizzazione dei percorsi commerciali/di trasporto e dell'Analisi del Ciclo di Vita degli aggregati. Gli aggregati sono scavati principalmente al di fuori dei Parchi Nazionali, siti Patrimonio dell'Umanità e altre aree protette. Attività d'estrazione illegali sono prevenute da sistemi efficienti di monitoraggio, sanzioni elevate e certificati di origine degli aggregati venduti. Le aree di salvaguardia dei minerali sono definite in modo che le ubicazioni note di risorse specifiche di minerali di importanza locale e nazionale non siano inutilmente sterilizzate da sviluppi non minerari, purché non creando una presunzione che le risorse definite verranno lavorate. In queste aree altre proposte di sviluppo non sono normalmente autorizzate. I terreni sfruttati sono risanati alla primissima 
opportunità svolta secondo elevati standard ambientali. La Pianificazione degli aggregati si occupa dell'aspetto finanziario del ripristino. L'implementazione delle Politiche di Pianificazione degli Aggregati è monitorata regolarmente.

\section{Altre politiche correlate}

Tutti i Paesi SEE hanno politiche relative agli aggregati (Politiche di Sviluppo Sostenibile, Politiche Ambientali, Politiche Minerali, Politiche di Gestione dei Rifiuti, Politiche di Recupero e Riciclaggio e Politiche di Pianificazione dell'Uso del Suolo) su un livello sviluppato in modo simile. Le risorse minerarie comprendenti gli aggregati sono considerate equivalenti alle altre risorse naturali del suolo. Le limitazione dell'uso del suolo sono diminuite rispetto al 2014. I Piani di Uso del Suolo sono redatti a livello nazionale, regionale e locale. Essi contengono le aree designate per l'estrazione e la protezione della risorse minerale, in questo modo la Pianificazione Minerale è una parte della Pianificazione dell'Uso del Suolo. L'esame della valutazione sostenibile è obbligatoria. La Valutazione Ambientale Strategica (VAS) è applicata come un esercizio collaterale anteriore alla pianificazione mineraria. L'estrazione in aree di conservazione naturale sono regolamentate basandosi sulle linee guida relative pubblicate dalla Commissione Europea nel 2010. Questo documento presenta come l'estrazione degli aggregati e gli obiettivi di biodiversità possono venire amministrati in armonia. Aree territoriale dove le risorse geologiche di aggregati esistono, sono assegnate nei piani di uso del suolo per tenere conto della futura estrazione di aggregati nell'ottica di evitare la sterilizzazione della risorsa. Le Politiche di Gestione dei Rifiuti promuovono il recupero, come aggregati, dei rifiuti minerari, di costruzione e demolizione, terre e rocce da lavori civili e dei rifiuti industriali. Non vi è alcun impedimento burocratico al recupero. La transizione da rifiuto a materiali primi secondi è incoraggiato; i rifiuti da costruzione e demolizione devono venire trasformati in riciclati almeno per il $70 \%$.

\section{Procedure di autorizzazione}

Il processo di autorizzazione per l'estrazione di aggregati primari è semplice, veloce ed efficace, guidato da un organismo regolatore considerevole. Le autorità coinvolte cooperano in modo efficiente. II modello di "sportello unico" è applicato, ed è la soluzione più vicina all'utenza. Sistemi efficienti autorizzativi forniscono l'accesso alle risorse grezze di materiale vicino alle piazze economiche più grandi, in tal modo ottimizzano anche l'efficienza del trasporto, insieme con la minimizzazione della sovraordinazione da parte dei clienti e con progetti efficaci degli usi finali che rimuovono qualsiasi spreco. Le scadenze del procedimento sono chiaramente regolamentati sia per le autorità che per gli utenti. I soggetti interessati sono coinvolti nel procedimento. L'amministrazione online (comprendendo moduli di domande in formato elettronico, osservazione automatica delle scadenze, documentazione digitale) è utilizza- 
ta durante il processo. A causa dei maggiori investimenti impegnati, le autorizzazioni estrattive devono essere solitamente di 10-20 anni per le cave di sabbia e ghiaia, e generalmente 20-50 anni per le cave di roccia da frantumazione, secondo la scala in entrambi i casi. L'uso di strumenti normativi finanziari progressivi a tempo (es. tasse di uso del suolo) difendono contro l'occupazione speculativa del terreno.

\section{Coinvolgimento dei portatori di interesse}

Il conflitto di diversi usi del suolo (es. aree estrattive e protette) è risolto per mezzo di consultazioni con le autorità, ONG, comunità locali e industria. I portatori d'interesse sono coinvolti nel processo di pianificazione. Il settori estrattivo coopera con autorità agricole ed ambientali. Le comunità locali beneficiano delle rendite derivanti da diritti minerari in tal modo sono interessati allo sfruttamento estrattivo.

\subsubsection{Livello regionale e locale}

Le autorità di pianificazione regionale e locale partecipano anche nella pianificazione attraverso la preparazione di piani regionale e locali basati sulla domanda regionale e locale. Per i piani locali sono preparati studi di base di valutazione del paesaggio locale e valutazioni d'impatto ambientale. Una strategia paesaggistica e piani di ripristino sono sviluppati fin dall'inizio considerando l'uso dopo la conclusione dell'estrazione del sito. Le autorità di pianificazione locale specificano i criteri ambientali per assicurare che le operazioni autorizzate non abbiano impatti sfavorevoli inaccettabili sull'ambiente naturale e storico-culturale o sulla salute umana.

\subsubsection{Problematiche Europee}

Una coerente politica comunitaria degli aggregati è sviluppata in modo che tenga in considerazione in modo equilibrato gli aspetti economici, ambientali e sociali per assicurare pratiche sostenibili dell'industria degli aggregati (Hámor et al. 2011). Una terminologia legale aggiornata per gli aggregati (sia primari che secondari) è usata e adottata dagli Stati membri. Marchi di qualità ecologica e legislazione eco-premianti specifiche per un prodotto sono estese sia agli schemi di produzione degli aggregati primari che secondari. Linee guida tecniche sono sviluppate in modo che includano direttive su come questa gestione dei rifiuti estrattivi corrisponda alla riserva di aggregati. La rete Natura 2000 è implementata in modo omogeneo nei diversi Stati Membri nell'ottica di evitare la deformazione delle condizioni di mercato, e lo spostamento fuori dai confini degli impatti ambientali.

\subsection{Problematiche ambientali}

Gli appalti pubblici sono in gran parte verdi, visto che utilizzano aggregati riciclati. L'Industria degli Aggregati affronta la gestione delle acque, del paesaggio e del suo- 
lo durante l'estrazione attraverso le migliori tecnologie disponibili e lo scambio di buon pratiche. Cave e fosse sfruttate sono ripristinate dopo l'utilizzo o convertite ad altri usi vantaggiosi per la comunità locale. La rete di siti degli aggregati contribuisce alla sviluppo di infrastrutture verdi, creando "trampolini di lancio" per la biodiversità. La Valutazione d'Impatto Ambientale (VIA): la procedura è semplificata, funziona come una procedura a sportello unico, meglio e con maggior aderenza tempistica alle procedure definite nella stessa direttiva VIA. La coordinazione fra VIA e altre direttive europee e politiche funziona propriamente, particolarmente con la VAS (Valutazione Ambientale Strategica) e AA (Valutaziona Appropriata).

\subsubsection{Inquinamento dell'aria}

Tenere l'inquinamento dell'aria sotto i limiti: le misure sono prese per ridurre la polvere e le emissioni di gas (per mezzo di sistemi di depolverizzazione che raccolgono gli scarti fini; usando sistemi d'aspirazione delle polveri nei mulini; coprendo i sistemi come i nastri trasportatori/di frantumazione e gli automezzi; mantenendo bagnate la strada o l'aree di cava). Polvere e emissioni di gas sono monitorate e valutate sistematicamente.

\subsubsection{Rumore}

Mantenere il rumore sotto i limiti: il rumore non necessario è eliminato e le emissioni di rumore sono ridotte (es. utilizzando sistemi di soppressione del rumore, spegnendo l'attrezzatura se non in uso, evitando non necessari fuori giri dei motori; usando rivestimenti di gomma negli scivoli, nei ribaltabili, nei camion, punti di trasferimento; usando appropriate tecniche di brillamento e materiale esplosivo; costruendo nuove strade per bypassare le aree residenziali). Un regolare monitoraggio del rumore è attivato e i risultati sono paragonati con i limiti di legge e con le condizioni ambientali specifiche del sito.

\subsubsection{Acque}

Per mantenere la qualità delle acque di falda: la gestione delle acque responsabile e sostenibile è assicurata da diversi mezzi, es. il piano di gestione delle acque (che si occupa sia di acque superficiali che profonde), riutilizzo dell'acqua e controllo di qualsiasi scarico dal sito. Le strutture di protezione della qualità delle acque sono usate (es. geotessile impermeabile per coprire l'area di manutenzione dell'attrezzatura, canali di drenaggio attorno all'area di sfruttamento) per evitare potenziali contaminazioni delle acque di falda. La qualità sia delle acque superficiali che profonde è monitorata. Studi idrogeologici sono condotti per osservare i cambiamenti della qualità delle acque. Durante il processo di ripristino si creano laghi e 
zone umide, le quali forniscono servizi eco sistemici come rifugi per biodiversità, gestione delle inondazioni e depurazione delle acque.

\subsubsection{Rifiuti minerari}

La gestione dei rifiuti prodotti è praticata durante l'intero ciclo degli scavi in cava. I rifiuti minerari sono usati come aggregati secondari o per l'architettura del paesaggio, ripristini o come materiale da colmatazione. Se il ripristino non è possibile, i cumuli di rifiuti sono immersi nel paesaggio e vegetati il prima possibile. La gestione dei rifiuti funziona secondo la gerarchia stabilita nella Direttiva Quadro Rifiuti: prevenzione della creazione del rifiuto, preparazione per il riutilizzo, riciclaggio, recupero e smaltimento come ultima possibilità.

\subsubsection{Biodiversità e compensazione ecologica}

Secondo il Documento Guida della Commissione Europea sui NEEl e Natura 2000 (EC 2010a, p. 74) le compensazioni di Biodiversità sono "risultati di conservazione misurabili derivanti da azioni studiate per compensare impatti significativi residui sfavorevoli alla biodiversità che sorgono dallo sviluppo del progetto dopo che una prevenzione appropriata e misure di mitigazione sono state intraprese. L'obiettivo delle compensazioni della biodiversità è di ottenere nessuna perdita netta e preferibilmente nessun guadagno netto di biodiversità sul posto secondo la composizione delle specie, della struttura dell'habitat, della funzione dell'ecosistema e l'uso della popolazione e i valori culturali associati con la biodiversità". Le attività di sfruttamento hanno sempre un impatto ambientale/paesaggistico che non può essere recuperato. $\mathrm{Ma}$, nonostante le compensazioni finanziarie o le tasse il ripristino è la migliore metodologia per l'industria degli aggregati di sviluppare una nuova biodiversità in un'area sfruttata. II recupero ambientale di un'area è ottenuto sia attraverso la mitigazione e il ripristino progressivo durante le fasi di scavo e la messa in pristino finale. Esso dovrebbe sempre essere la via preferita per fermare la perdita di biodiversità, e le compensazioni considerate come un'ultima risorsa per riparare gli impatti residui inevitabili. Il ripristino è implementato in un contesto paesaggistico, prendendo in considerazione valori biologici, sociali e culturali.

\subsubsection{Ripristini}

Prima del ripristino viene sviluppata una strategia di recupero ambientale globale, che individui gli usi successivi proposti del sito. Essa contiene informazioni riguardo le risorse del terreno e l'idrologia, e su come il terreno superficiale/sottosuolo/di copertura per fare materiali sono da trattare finché l'estrazione ha luogo; una valutazione del grado di classificazione dei terreni agricoli; e una strategia paesaggistica. II ripristino comincia dall'inizio degli scavi, e questo processo continuo di recupero 
ambientale deve essere concorde con il piano di ripristino finale, rendendo il processo più semplice e più efficace. Un approccio equilibrato è adottato fra conservazione della natura e attività estrattive. I piani di ripristino sono discussi prima dell'attuazione con i soggetti interessati locali. II piano di ripristino sono adattati in anticipo secondo l'uso potenziale successivo del sito di cava dopo la chiusura (se specificato nei piani di sviluppo locali). Quando il piano di recupero è relativo al cambiamento dell'uso del suolo, nuove strutture sono create come laghi in casi di cave profonde con fondo impermeabile o strutture sportive o parchi ricreazionali o zone umide. Le caratteristiche naturali (es. ruscelli) sono recuperati e vivai sono sviluppati durante il periodo di funzionamento della cava per facilitare la fase di ripristino con specie locali. II terreno degradato è recuperato attraverso l'utilizzo o del terreno superficiale rimosso e conservato durante la fase iniziale di coltivazione della cava o persino di altro terreno superficiale proveniente da altre aree. Si previene la contaminazione residua.

\subsection{Problematiche sociali}

Un coinvolgimento potenziato ed evoluto della società locale è assicurato nei Paesi SEE dallo Stato o dalle società di aggregati su base volontaria. In questo modo l'accettazione sociale dell'estrazione di aggregati è garantita dalla fase di pianificazione. I soggetti interessati locali sono informati in modo obiettivo ed educati in merito agli aggregati primari e secondari attraverso, es. una seria di incontri, lavori di gruppo, sessioni d'ascolto, mappe di uso del suolo, newsletter, volantini e poster. Le esigenze delle comunità locali sono considerate e rispettate sia dalle autorità della pianificazione regionale che dalle imprese di scavo degli aggregati. Le comunità locali sono consapevoli dei piani minerali locali, e della disponibilità e dei requisiti delle risorse minerali. Le imprese di aggregati impiegano lavoratori locali.

\subsection{Problematiche economiche}

La dipendenza dall'import è stata ridotta nei Paesi SEE grazie a politiche minerali multidisciplinari sia per materiali grezzi minerali per la produzione energetica che non, comprendendo gli aggregati primari e secondari. In aggiunta la produzione e l'export degli aggregati sono promossi per mezzo del miglioramento delle condizioni di approvvigionamento dall'Unione Europea, diversificando le fonti di materiali grezzi e migliorando l'efficienza della risorsa (compreso il riciclaggio) e trovando materiali grezzi alternativi. La valutazione delle economie di aggregati risiede nel concetto di SSM - Sustainable Supply Mix (Mix di fornitura sostenibile). L'intero ciclo di vita delle cave, comprendente l'esplorazione, l'estrazione, il trattamento e il ripristino dei siti, è pianificato in modo globale e multidisciplinare. 


\subsubsection{Politiche transnazionali SARM}

L'analisi delle strutture transnazionali del mercato degli aggregati è regolare. La domanda e la fornitura (fonte) sono ben note e pubblicate, così come gli scenari di medio e lungo periodo della domanda e le opzioni possibili di approvvigionamento. Sia la domanda che l'approvvigionamento di aggregati possono essere influenzati dalle politiche transnazionali SARM (che a turno devono essere basate su tali analisi di mercato). Il quadro delle politiche transnazionale SARM è attuato nei Paesi SEE per soddisfare la crescente domanda di aggregati, in particolare nei centri urbani maggiori localizzati in aree di confine. Nel quadro di gare d'appalto internazionali riguardanti la costruzione (transfrontaliera) di strade e ferrovie, il fabbisogno di aggregati e di logistica per il trasporto è chiaramente definito. La maggior parte degli strumenti finanziari sono all'interno del dominio della sovranità nazionale. È compito del governo stabilire questi elementi trainanti economici insieme alla politica nazionale; tuttavia, differenze significative negli strumenti finanziari possono portare ad una competizione distorta nelle regioni transfrontaliere. Strumenti finanziari sono armonizzati nei Paesi SEE. Lo scambio di dati e conoscenze è migliorato fra i soggetti interessati tenendo conto del livello delle politiche transnazionali. Le consultazioni dei soggetti interessati sono regolari, dibattiti almeno annuali sono efficaci. Il quadro politico SARM è attuato nella rete di trasporto transnazionale europea: la relazione fra "aggregati e costruzioni" a livello transnazionale è presa in considerazione, includendola nelle obiettivi e strategie ERDF.

\subsubsection{Tassazione}

Le tasse sullo smaltimento dei rifiuti da C\&D promuovono lo spostamento del consumo dagli aggregati primari ai secondari. Le misure finanziarie sono istituite nell'ottica di supportare il recupero dei rifiuti da costruzione e demolizione. La tassazione delle discariche promuove la minimizzazione del rifiuto e il recupero. Lo scopo delle tasse è di esortare le imprese ed i consumatori a produrre meno rifiuti, a scoraggiare le discariche ed a spronare la minimizzazione dei rifiuti e gli investimenti in altre forme di recupero di materiale e/o di risorsa. Imposta aggregati: per proteggere la competitività internazionale le tasse sono quindi imposte sulle importazione ma le esportazioni sono esonerate. L'imposta è intesa affrontare i costi ambientali associati con le operazioni estrattive (rumore, polvere, intrusione visiva, perdita di comfort e danno alla biodiversità) in linea con la dichiarazione del Governo di intento sulla tassazione ambientale. Il suo obiettivo è di ridurre la domanda di aggregati nuovi e incoraggiare l'uso di materiali riciclati e aggregati secondari come il rifiuto di caolino, di ardesia e rifiuti minerari dall'estrazione del carbone, che sono esenti [dalle tasse]. Basandosi sul fatto che la tassazione sull'estrazione di materiali primari o sull'uso 
della risorsa non è uno strumento appropriato per incrementare l'efficienza della risorsa stimoli positivi aiutano enormemente l'industria degli aggregati.

\subsection{Attività professionali}

\subsubsection{Inventario}

Inventari completi di aggregati primari e secondari sono sviluppati nei Paesi SEE includendo l'attività mineraria inerte e i rifiuti da costruzione e demolizione o che hanno accesso fra loro. L'inventario completo delle risorse e riserve di aggregati primari e dei dati di produzione consiste in dati spaziali relativi alle dimensioni e forma delle zone di sfruttamento, delle riserve comprovate, tipo e qualità degli aggregati. L'inventario degli aggregati secondari contiene informazioni sulle caratteristiche tecniche (tipo, quantità, trattamento, metodo, ecc.), posizione, impianti di recupero, standard tecnici ecc. L'inventario richiede investimenti aggiuntivi per lo staff e la formazione. Le conoscenze rilevanti riguardanti i materiali grezzi in Europa sono ottenute e tutti i portatori di interesse, compresi le autorità pertinenti, le imprese di materiali grezzi e quelle consecutive all'estrazione, le comunità di ricerca e la società lavorano per gli stessi obiettivi. Nuove banche dati affidabili (collegate al potenziale minerale europeo) sono costruite per nutrire la base di conoscenza europea sui materiali grezzi primari e secondari in un modo armonizzato e standardizzato. Lo scopo è di ingrandire la base conoscitiva europea nell'ottica di migliorare la fornitura sostenibile e sicura dei materiali grezzi all'economia e società europea, che include: (a) la costruzione di una base conoscitiva innovativa delle risorse europee, sia primaria che secondaria e (b) il miglioramento della quantità e qualità dell'istruzione superiore (esperti qualificati) insieme con la ricerca e l'impresa in Europa per l'intera filiera dei materiali grezzi. Un sistema di modellazione dinamica per i materiali primari e secondari è sviluppato e comunemente utilizzato nella pianificazione degli aggregati. Inventari: online, servizi facilmente accessibili (o persino disponibili pubblicamente) che forniscono informazioni sulle fonti di fornitura degli aggregati sia primari che secondari (e domanda), sono necessari in tutti i Paesi. Basandosi sulla competenza professionale necessaria, sulle banche dati esistenti e sulle attività normative, i servizi geologici e le autorità minerarie riescono a far funzionare tale sistema. Grazie a altre prassi in certi Paesi, vi è buona cooperazione fra servizi geologici e autorità minerarie e autorità di pianificazione regionale e enti ambientali statali. I ministeri nazionali accettano l'importanza dell'acquisizione di statistiche più affidabili e complete sugli aggregati.

\subsubsection{Geologia e potenziale degli aggregati}

La valutazione della presenza di sabbia e ghiaia è stata basata sulla mappa compilata dei sedimenti non consolidati. Essa mostra sia la distribuzione regionale delle unità geologiche di sedimenti non consolidati e le loro facies, mentre fornisce informazioni 
in merito alle caratteristiche litologiche più importanti del materiale. La classificazione geologica della presenza di roccia frantumata e i depositi è condotta sulla base di mappe geologiche. La qualità e quantità dei materiali grezzi e l'importanza della presenza per gli approvvigionamenti locali o regionali sono presi in considerazione. Tutti questi dati sono gestiti in un inventario GIS con accesso online da chiunque.

\subsubsection{Salvaguardia minerale}

Il concetto di aree di salvaguardia minerale è ampiamente conosciuto e attuato. "la presenza di una MSA [Mineral Safeguarding Area] né preclude altre forme di sviluppo dall'essere permesse né trasmette alcuna presunzione che il minerale sarà estratto. MSA semplicemente provvede uno strumento politico che sarà un campanello di allarme al fatto che i minerali possono essere sterilizzati dallo sviluppo non-minerale proposto e che ciò dovrebbe essere considerato dal processo di pianificazione..." (Wrighton et al. 2011).

\subsection{Tecnologia}

L'industria degli aggregati utilizza le migliori tecnologie disponibili (BAT - Best Available Technologies) per assicurare l'estrazione più efficiente e tecnologie verdi al grado più alto possibile, minimizzando la sua impronta territoriale e gli impatti ambientali che comprendono l'uso delle acque, la preservazione e l'incoraggiamento della biodiversità sia nelle cave e fosse operative che ripristinate.

\subsubsection{Recupero/Riciclaggio}

I materiali grezzi secondari, come i rifiuti minerari, i rifiuti da costruzione e demolizione, le terre e rocce da scavi civili e i rifiuti industriali, sono utilizzati come aggregati. I rifiuti da costruzione e demolizione (C\&D) sono recuperati almeno per il $70 \%$ come è prescritto dalla Direttiva 2008/98/CE. II recupero di C\&D offre importanti opportunità: riduce il bisogno di terreno per le discariche (i); impedisce il sovraconsumo di risorse naturali di aggregati non-rinnovabili, grazie all'introduzione di materiali alternativi e aggiuntivi sul mercato degli aggregati (ii); crea nuove opportunità di affari dal riciclaggio dei rifiuti (iii). I rifiuti da C\&D sono preordinati in categorie alla fonte in parti omogenee. Questa demolizione selettiva riduce i costi di recupero o smaltimento (dove applicata) ed assicura un migliore qualità dei prodotti riciclati. Gli aggregati riciclati sono ampiamente utilizzati in applicazioni non legate di grandi quantitativi per opere infrastrutturali (fondazioni di strade e ferrovie), per ripristini ambientale e per la preparazione di misture di calcestruzzo basso resistenti. La scelta di aggregati per una specifica applicazione dipende solo dalle caratteristiche del materiale e non dalla sua origine. Le norme tecniche europee di utilizzo non distinguono gli aggregati in funzione della loro origine ma secondo le loro caratteristiche. In tal modo i prodotti aggregati riciclati che soddisfano le norme europee predominanti e 
le specifiche tecniche e sono marchiati $\mathrm{CE}$, possono competere con gli aggregati convenzionali. L'uso integrato di aggregati naturali e riciclati, oltre a salvare apprezzabilmente le risorse naturali, permette un miglior sfruttamento delle risorse disponibili secondo i differenti usi.

\subsubsection{Esplosivi}

L'industria degli aggregati utilizza esplosioni sequenziali e tecnologia moderna per ridurre le vibrazioni e il rumore che sono monitorati. Se le naturali circostanze permettono metodi di estrazioni alternativi, l'uso dell'esplosivo viene evitato.

\subsubsection{Sicurezza}

Le imprese di aggregati prestano attenzione alla salute e sicurezza, focalizzandosi sul Danno Zero prevenendo morte, lesioni e cattiva salute nell'ambiente di lavoro. Ciò richiede non solo dipendenti completamente competenti e sicuri, ma che tutti gli elementi della filiera lavorino insieme per assicurare che i lavoratori siano provvisti con i dispositivi e equipaggiamenti di sicurezza che soddisfino i requisiti della guida SAFER BY DESIGN.

\subsection{Trasporti}

L'efficienza massima del trasporto è ottenuta attraverso la pianificazione strategica delle reti ferroviarie e le vie d'acqua cosicché più aggregati sono trasportati da questi mezzi. Le politiche nazionali di pianificazione degli aggregati sono integrate con le strategie di sviluppo del trasporto ferroviario e fluviale/marino per assicurare la fornitura ottimale di aggregati e soluzioni di trasporto per governi, l'industria, operatori del trasporto e società civile. II trasporto su strada è ottimizzato per mezzo di accessi migliorati alle risorse il più vicini possibile ai mercati più grandi. Le distanze di trasporto sono ridotte dall'uso di risorse locali. Questo minimizza anche le emissioni di $\mathrm{CO}_{2}$ relative e il consumo di energia, e allo stesso modo l'impatto ambientale e la congestione del trasporto. L'uso di camion con carico utile maggiore riduce ulteriormente il numero di movimenti di automezzi, uso di carburante e le emissioni $\mathrm{CO}_{2}$. il costo di trasporto della sabbia e ghiaia lungo una distanza di più di $30 \mathrm{~km}$ eccede il valore del materiale grezzo, in tal modo l'efficienza dei costi di trasporto è ottenuta dall'uso di risorse locali di aggregati. 


\section{VISIONE CONGIUNTA}

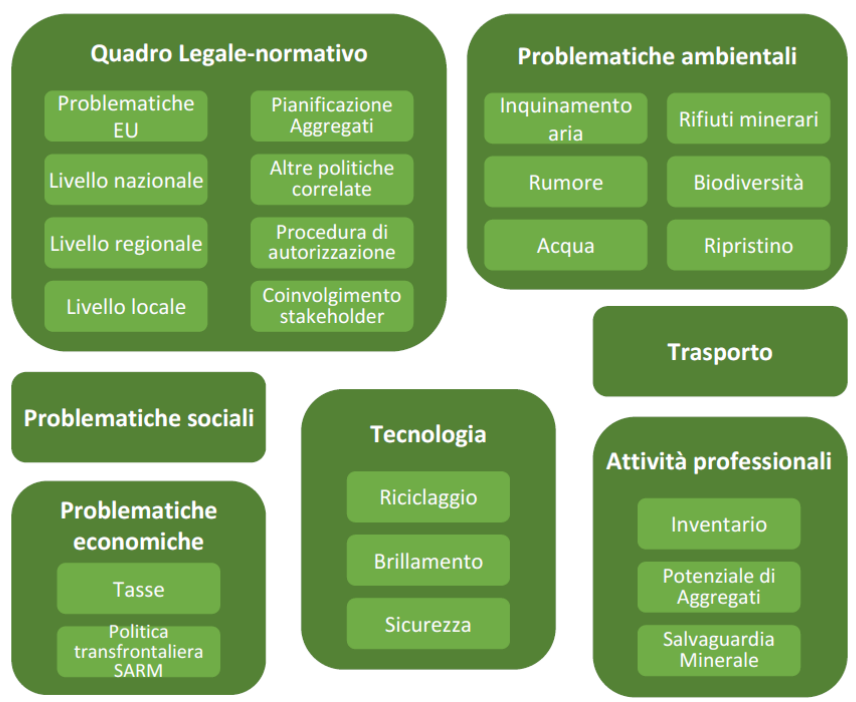

Figura 4.2. Argomenti trattati riguardanti la Visione Congiunta 


\section{Raccomandazioni sulle Migliori Pratiche nella Pianificazione degli Aggregati}

Come un resoconto, le raccomandazioni sono raccolte per ognuno dei capitoli. Forniscono le dichiarazioni più importanti che è necessario considerare quando si inizia o si gestisce una pianificazione degli aggregati completa ed integrata.

Le raccomandazioni sono basate sui risultati dell'analisi multisettoriale, alcune buon pratiche di pianificazione degli aggregati e sull'esperienza dei partner del progetto SNAP-SEE comprendendo le consultazioni che hanno tenuto. Incoraggiamo $i$ lettori a distribuire questo manuale e a considerare di seguire $i$ passi collaudati verso l'istituzione di una pianificazione degli aggregati sostenibile nel Sud Est Europa. 


\section{Pianificazione degli aggregati}

$\checkmark \quad$ Le Politiche di Pianificazione degli Aggregati sono richieste per assicurare la fornitura sostenibile degli aggregati. In queste politiche di pianificazione gli aggregati primari e secondari dovrebbero essere gestiti insieme per proteggere le risorse primarie e ridurre i volumi dei rifiuti minerari e da C\&D, oltre che i sottoprodotti industriali. Nell'ottica di garantire l'accesso alla risorsa di aggregati, i Piani degli Aggregati devono guardare al futuro almeno di 20 anni e dovrebbero essere aggiornati almeno ogni 5-10 anni.

$\checkmark \quad$ La Pianificazione degli Aggregati dovrebbe essere attuata a livelli nazionali, regionali e locali. La pianificazione nazionale è un approccio generale, mentre i piani regionali e locali sono dettagliati.

$\checkmark$ Durante lo sviluppo delle Politiche di Pianificazione degli Aggregati, si dovrebbero coinvolgere i soggetti interessati rilevanti; in questo modo le Politiche attraversano una procedura di consultazione pubblica.

\section{Procedura d'autorizzazione}

$\checkmark \quad$ II processo d'autorizzazione per l'estrazione di aggregati primari dovrebbe essere semplice, veloce ed efficace, guidato da un ente regolatore considerevole. II modello a "sportello unico" sembra essere la soluzione più vicina all'utente e può avere la migliore efficienza nell'amministrazione burocratica. A causa dei maggiori investimenti interessati, le autorizzazioni all'estrazione devono essere solitamente di 10-20 anni per cave di sabbia e ghiaia, e normalmente di 20-50 anni per le cave di roccia frantumata.

\section{Problematiche sociali}

$\checkmark$ Un coinvolgimento potenziato ed evoluto della società locale è assicurato nei Paesi SEE dallo Stato o dalle imprese di aggregati su base volontaria.

$\checkmark \quad$ I conflitti sui differenti usi del suolo dovrebbero diminuire grazie alle consultazioni dei soggetti interessati più rilevanti (es. decisori, autorità ambientali, industria estrattiva, ONG).

\section{Problematiche ambientali}

$\checkmark \quad$ La rete Natura 2000 si dovrebbe attuare in modo omogeneo nei diversi Stati Membri per evitare la deformazione delle condizioni di mercato, e l'esportazione transfrontaliera degli impatti ambientali.

$\checkmark \quad$ L'Industria degli Aggregati affronta gestione delle acque, gestione dei terreni e del paesaggio durante l'estrazione degli aggregati per mezzo delle migliori tecnologie disponibili e lo scambio di buone pratiche. 


\section{Bibliografia}

Bressi, G., Volpe, G., Pavesi, E. (2011). The production of recycled aggregates. SARMa Manual.

Chalkiopoulou, F., Hatzilazaridou, K. (2011). How to Achieve Aggregates Resource Efficiency in Local Communities. SARMa Manual.

Chalkiopoulou, F., Hatzilazaridou, K. (2013). How is Planning currently being carried out in Greece? SNAP-SEE project, www.snapsee.eu (WP5.1 report)

Cibin et al. (2011). Providing a Sustainable Supply Mix of Aggregates: State-of-the-art in South-East Europe.

Department for Communities and Local Government (2012). 'National Planning Policy Framework' (www.communities.gov.uk).

Department for Communities and Local Government (2014). ‘Planning Practice Guidance' (http://planningguidance.planningportal.gov.uk).

European Commission (2010a). EC Guidance on undertaking new non-energy extractive activities in accordance with Natura 2000 requirements

European Commission (EC), DG Enterprise and Industry (2010b): Abridged report of the ad-hoc Working Group on Exchanging Best Practice on Land Use Planning, Permitting and Geological Knowledge Sharing

Hámor, T. (2004). Sustainable mining in the European Union: The legislative aspect Environmental Management, Vol. 33., pp. 252-261.

Hámor, T. (2011). Synthesis report on legislation and regulatory framework related to sustainable aggregate resources management in selected South East European Countries - SARMA project, Budapest, $156 \mathrm{p}$.

Hámor, T., Tiess, G., Kager, J., Heimburg, J. (2011). European Community law relevant to aggregates - SARMA project, Budapest, $46 \mathrm{p}$.

Hicks, L. (2008). Aggregates supply in England. Issues for planning. - British Geological Survey Open Report, OR/08/059. 24 pp.

Horváth, Z., Sári, K. (2013). How is Planning currently being carried out in Hungary? SNAP-SEE project, www.snapsee.eu (WP5.1 report)

Horváth, Z., Sári, K. (2014a). Multi-sectoral Analysis of State of Planning Aggregates. SNAP-SEE project, www.snapsee.eu (WP5.1 report)

Horváth, Z., Sári, K., (2014b). Guidance for Aggregates Planning Questionnaire for Hungary, SNAP-SEE project, www.snapsee.eu (WP5.2 report) 
Janova, V., Fabian, M., Mesarcik, I. (2013). How is Planning currently being carried out in Slovakia? SNAP-SEE project, www.snapsee.eu (WP5.1 report)

Maruntiu, M., Coltoi, O., Marica, S. (2013). How is Planning currently being carried out in Romania? SNAP-SEE project, www.snapsee.eu (WP5.1 report)

Maruntiu, M., Marica, S., Coltoi, O., (2014). Guidance for Aggregates Planning Questionnaire for Romania, SNAP-SEE project, www.snapsee.eu (WP5.2 report)

Mati, S., Moisiu, L., Avxhi, A. SH.GJ.SH (2013). How is Planning currently being carried out in Albania? SNAP-SEE project, www.snapsee.eu (WP5.1 report)

Miko, S., Kruk, B., Dedić, Ž., Kovačević Galović, E., Hasan, O. (2013). How is Planning currently being carried out in Croatia? SNAP-SEE project, www.snapsee.eu (WP5.1 report)

Miko, S., Kruk, B., Dedić, Ž., Kovačević Galović, E., Hasan, O., (2014a). Guidance for Aggregates Planning Questionnaire for Croatia, SNAP-SEE project, www.snapsee.eu (WP5.2 report)

Miko, S., Kruk, B., Dedić, Ž., Kovačević Galović, E., Hasan, O. (2014b). Guidance on Aggregates Planning in South East Europe. SNAP-SEE project, www.snapsee.eu (WP5.2 report)

Miletić, S., Žibret, G., Burger, A. (2013). How is Planning currently being carried out in Slovenia? SNAP-SEE project, www.snapsee.eu (WP5.1 report)

Miletić, S., Žibret, G., Senegačnik, A., Rokavec, D., (2014). Guidance for Aggregates Planning Questionnaire for Slovenia, SNAP-SEE project, www.snapsee.eu (WP5.2 report)

Moisiu, L., Mati, S. (2014). Guidance for Aggregates Planning Questionnaire for Albania, SNAP-SEE project, www.snapsee.eu (WP5.2 report)

Moltrer, A., Valbusa, M. (2013). How is Planning currently being carried out in Autonomous Province of Trento (Italy)? SNAP-SEE project, www.snapsee.eu (WP5.1 report)

Moltrer, A., Valbusa, M. (2014). Guidance for Aggregates Planning Questionnaire for Trento - Italy, SNAP-SEE project, www.snapsee.eu (WP5.2 report)

Ontario Ministry of Natural Resources (2010). The State of the Aggregate Resource in Ontario Study. Consolidated Report

(http://www.mnr.gov.on.ca/stdprodconsume/groups/lr/@mnr/@aggregates/do cuments/document/286996.pdf)

Poulin, R., Bilodeau, M.L. (1993). A model of a mineral aggregate market: The Eastern Coastal USA. Resources Policy, Volume 19, Issue 2. 
Radusinovic, S., Čović, R., Asanović, D. (2013). How is Planning currently being carried out in Montenegro? SNAP-SEE project, www.snapsee.eu (WP5.1 report)

Rizzati, A., Romagnoli, M., Marasmi, C. (2013). How is Planning currently being carried out in Emilia-Romagna Region? SNAP-SEE project, www.snapsee.eu (WP5.1 report)

SARMa Glossary, http://www.sarmaproject.eu/index.php?id=1904

Shields, D.J., Solar, S.V. (2006). The nature and evolution of mineral supply choices. In: Cardu, M., Ciccu, R., Lovera, E. and Michelotti E. Proceedings of the 15th International Symposium on Mine Planning and Equipment Selection, September 20-22, 2006, Torino, IT. Galliate, IT: FIORDO, pp. 902-907.

Shields, D.J. (2013). Planning for Aggregates in the United Kingdom and Canada: strengths and weaknesses. SNAP-SEE project, www.snapsee.eu (WP6.1 report)

Simić V., Životić D., Andrić N., Miladinović Z. (2013). How is Planning currently being carried out in Serbia? SNAP-SEE project, www.snapsee.eu (WP5.1 report)

Simić, V., Životić, D., Andrić, N., Miladinović, Z., (2014). Guidance for Aggregates Planning Questionnaire for Serbia, SNAP-SEE project, www.snapsee.eu (WP5.2 report)

Strategic Implementation Plan For The European Innovation Partnership On Raw Materials (2013).

Susa, I., Broceta, V. (2013). How is Planning currently being carried out in Herzegbosnian Canton (Bosnia and Herzegovina)? SNAP-SEE project, www.snapsee.eu (WP5.1 report)

Suša, I., Broćeta, V., Kaselj, R., (2014). Guidance for Aggregates Planning Questionnaire for Herzegbosnian Canton (Bosnia and Herzegovina), SNAP-SEE project, www.snapsee.eu (WP5.2 report)

Tiess, G. (2010): Planning Policies and Permitting Procedures to Ensure the Sustainable Supply of Aggregates in Europe, Final Report, Leoben, 53. p.

Tiess, G. (2011): General and International Mineral Policy, Springer ISBN 978-3-211$89004-2,630 \mathrm{p}$.

Tiess, G., Chalkiopoulou, F. (2011). Sustainable Aggregates Resource Management and Sustainable Supply Mix at the Regional, National and Transnational Level. SARMa Manual. 61 p.

Tiess, G. (2013). How is Planning currently being carried out in Austria? SNAP-SEE project, www.snapsee.eu (WP5.1 report) 
Tiess, G. (2014). Guidance for Aggregates Planning Questionnaire for Austria; SNAPSEE project, www.snapsee.eu (WP5.2 report)

UEPG Position Paper: UEPG Key Messages on the EU Roadmap: Towards a resourceefficient Europe. www.uepg.eu

UEPG Position Paper: UEPG Sustainable Transport Policy. www.uepg.eu

UEPG Position Paper: Safer by Design. www.uepg.eu

UEPG Position Paper: Land Use and the Aggregates Industry. www.uepg.eu

UEPG Position Paper: UEPG Position on Resource Taxation. www.uepg.eu

UEPG Position Paper on Ecological Offsets. www.uepg.eu

UEPG Position Paper: Water Management in the Aggregates Industry. www.uepg.eu

Wrighton, C.E., McEvoy, F.M. and R. Bust (2011). Mineral Safeguarding in England: good practices advice. British Geological Survey Open Report OR/11/046. 53p. 



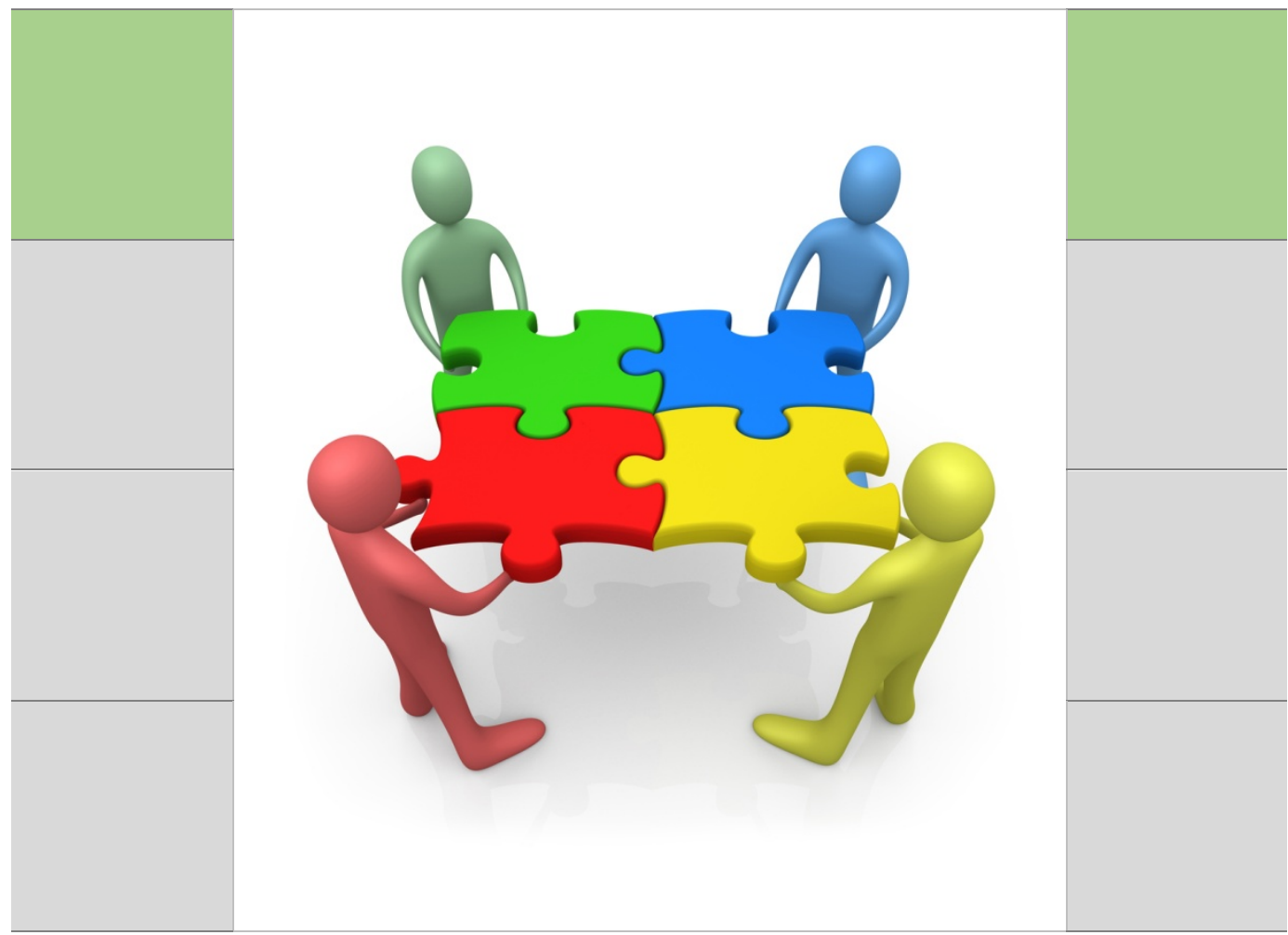

Sustainable Aggregates Planning in South East Europe (SNAP-SEE)

http://www.snapsee.eu 